\title{
Ordinary differential equations and descriptive set theory: uniqueness and globality of solutions of Cauchy problems in one dimension
}

\author{
by
}

Alessandro Andretta and Alberto Marcone (Torino)

\begin{abstract}
We study some natural sets arising in the theory of ordinary differential equations in one variable from the point of view of descriptive set theory and in particular classify them within the Borel hierarchy. We prove that the set of Cauchy problems for ordinary differential equations which have a unique solution is $\boldsymbol{\Pi}_{2}^{0}$-complete and that the set of Cauchy problems which locally have a unique solution is $\boldsymbol{\Sigma}_{3}^{0}$-complete. We prove that the set of Cauchy problems which have a global solution is $\boldsymbol{\Sigma}_{4}^{0}$-complete and that the set of ordinary differential equations which have a global solution for every initial condition is $\Pi_{3}^{0}$-complete. We prove that the set of Cauchy problems for which both uniqueness and globality hold is $\boldsymbol{\Pi}_{2}^{0}$-complete.
\end{abstract}

This paper deals with descriptive set-theoretic questions in the theory of ordinary differential equations (ODEs). Descriptive set theory (DST, from now on) is, roughly, the study of definable sets in Polish (i.e. separable completely metrizable) spaces. Definable means here: being $\mathbf{F}_{\sigma}, \mathbf{G}_{\delta}$, Borel, analytic, or, more generally, belonging to a "well behaved" collection of sets. The roots of this subject go back to the work of the analysts of the turn of the century: Borel, Lebesgue, and Baire in France and Lusin, Suslin, and Novikov in Russia. After the '50s DST was revolutionized by the techniques of mathematical logic: these allowed to solve long standing problems and changed the perspective of the subject (see [7] and [4] for more on the history and development of DST).

One of the main trends of current research (see [1] and Sections 23, 27, 33 and 37 of [5]) is the classification of natural sets arising in various parts of analysis, topology, etc. A couple of clarifications are in order. To classify here means to pin down the exact complexity of a given set: e.g. to show

1991 Mathematics Subject Classification: Primary 04A15; Secondary 34A12.

We thank our colleagues Anna Capietto and Camillo Costantini for helpful conversations. 
that a set $A$ is $\mathbf{F}_{\sigma}$ but not $\mathbf{G}_{\delta}$, or that it is analytic (i.e. a continuous image of a Borel set), but not Borel. The second clarification is about the concept of being a natural set. This means that the set comes up in actual mathematical practice, rather than being constructed in an ad hoc way - of course, this has to do more with the sociology of mathematics than with mathematics itself.

In this paper we study the complexity of the sets of all Cauchy problems for ODEs that have a unique solution, and the ones that have a global solution. (We think that both these sets are natural.) More precisely: given a continuous function $F: \mathbb{R}^{2} \rightarrow \mathbb{R}$ and $(x, y) \in \mathbb{R}^{2}$, consider the following Cauchy problem:

$$
\frac{d \varphi}{d t}=F(t, \varphi(t)), \quad \varphi(x)=y .
$$

Here and below we denote by $C\left(\mathbb{R}^{2}\right)$ the set of real-valued continuous functions on $\mathbb{R}^{2}$ with the compact-open topology. Thus $C\left(\mathbb{R}^{2}\right)$ is a Fréchet space. Then the set $\mathcal{U}$ of $(F, x, y)$ such that there exists a unique solution is a dense $\mathbf{G}_{\delta}$ in $C\left(\mathbb{R}^{2}\right) \times \mathbb{R}^{2}$. Therefore $\mathcal{U}$ itself is a Polish space. The set $\mathcal{U}_{\forall}$ of all $F$ 's such that for every $(x, y) \in \mathbb{R}^{2}$ the solution is unique is also a dense $\mathbf{G}_{\delta}$. (Both statements are true also in the case when $F$ varies in $C_{\mathrm{c}}\left(\mathbb{R}^{2}\right)$, the space of all functions constant at infinity, which is a Banach space; in this case we denote by $\mathcal{U}^{\mathrm{c}}$ and $\mathcal{U}_{\forall}^{\mathrm{c}}$ these sets.) The set $\mathcal{L U}^{\mathrm{c}}$ of $(F, x, y)$ such that the solution is locally unique is $\mathbf{G}_{\delta \sigma}$ (i.e. $\left.\boldsymbol{\Sigma}_{3}^{0}\right)$. The set $\mathcal{G}$ of $(F, x, y)$ such that there exists a global (i.e. defined on all of $\mathbb{R}$ ) solution is $\mathbf{F}_{\sigma \delta \sigma}$ (i.e. $\boldsymbol{\Sigma}_{4}^{0}$ ). The set $\mathcal{G}_{\forall}$ of all $F$ 's such that for every $(x, y) \in \mathbb{R}^{2}$ there exists a global solution is $\mathbf{F}_{\sigma \delta}$ (i.e. $\left.\boldsymbol{\Pi}_{3}^{0}\right) . \mathcal{G} \cap \mathcal{U}$ is $\mathbf{G}_{\delta}$ both in $C\left(\mathbb{R}^{2}\right) \times \mathbb{R}^{2}$ and in $\mathcal{U}$; similarly, $\mathcal{G}_{\forall} \cap \mathcal{U}_{\forall}$ is $\mathbf{G}_{\delta}$ both in $C\left(\mathbb{R}^{2}\right)$ and in $\mathcal{U}_{\forall}$.

It is worth noticing that the natural sets which appear for the first time in the Borel hierarchy at the fourth level are quite rare (and are usually $\boldsymbol{\Pi}_{4}^{0}$-complete), while there are no natural examples which appear for the first time at the fifth level (see [5], Section 23). To the best of our knowledge $\mathcal{G}$ is the first example of a natural set which is $\boldsymbol{\Sigma}_{4}^{0}$-complete (and thus Borel but not $\left.\Pi_{4}^{0}\right)$.

Since all the sets mentioned above are contained in a topological vector space $\left(C\left(\mathbb{R}^{2}\right), C_{\mathrm{c}}\left(\mathbb{R}^{2}\right)\right.$, or their product with $\left.\mathbb{R}^{2}\right)$ it is natural to ask whether they inherit the vector space structure. It will follow from our results that none of them (as well as their sections such as $\mathcal{U}_{(x, y)}$, the set of elements of $C\left(\mathbb{R}^{2}\right)$ such that $\left.(F, x, y) \in \mathcal{U}\right)$ does. Actually, it follows from standard descriptive set-theoretic results (and from our classification results) that none of them is even a group.

We will confine ourselves to ODEs in one dimension but it will follow from our proofs that the results regarding uniqueness we obtain can be extended without any effort to finite systems of ODEs, i.e. to ODEs in 
finite-dimensional Banach spaces. In contrast with this, the proofs of our upper bounds for the complexity of $\mathcal{G}$ and $\mathcal{G}_{\forall}$ rely heavily on the order structure of $\mathbb{R}$ and hence admit no obvious generalizations to the finitedimensional case. Indeed, the analogues of $\mathcal{G}$ and $\mathcal{G}_{\forall}$ in dimension two are respectively $\boldsymbol{\Sigma}_{1}^{1}$-complete and $\boldsymbol{\Pi}_{1}^{1}$-complete and hence not Borel. These results will be proved in a forthcoming paper. Similarly, considering ODEs in infinite-dimensional Banach spaces leads to several new difficulties (e.g. the failure of the Cauchy/Peano existence theorem) which we will not tackle in this paper.

Several papers have dealt with the uniqueness problem for regular ODEs as well as ODEs in Banach spaces, equations with a non-continuous $F$, and functional differential equations ([8], [6], [3]) from a viewpoint somewhat similar to ours. The paper of Orlicz shows that $\mathcal{U}_{\forall}^{c}$ is comeager when we restrict ourselves to bounded continuous F's. By inspecting the proof one can see that it shows also that the set is $\mathbf{G}_{\delta}$ dense. The other papers deal with larger function spaces but they focus on Baire category and prove only that the appropriate analogue of $\mathcal{U}_{\forall}$ is a comeager set-it contains a dense $\mathbf{G}_{\delta}$ set. Our other results do not seem to have siblings in the existing literature.

ODEs have been studied with the techniques of mathematical logic from a different viewpoint by Simpson in [9]: the goal there was to classify the Cauchy/Peano and the Ascoli-Arzelà theorems according to their axiomatic strength.

We tried to make the paper accessible to both logicians and analysts. In Section 1 we briefly introduce the terminology and techniques of DST that will be used in the sequel. The reader who already knows the basics of the subject can safely skip this section and refer back to it when needed. In Section 2 we start to deal with ODEs by introducing some techniques and proving some basic facts that will be used several times in the rest of the paper. Sections 3, 4 and 5 study the complexity of sets related to three of the basic properties of Cauchy problems for ODEs, respectively uniqueness of solutions, local uniqueness of solutions and existence of solutions defined on the whole real line.

1. Descriptive set theory. The purpose of this section is to get the reader acquainted with some standard concepts and results of DST which will be used in the rest of the paper. All the facts that will be quoted without proof can be found in [5].

1.1. Polish spaces. A topological space $X$ is Polish if it is separable and completely metrizable, i.e. admits a compatible complete metric. For example separable Banach spaces, metrizable compact spaces, countable discrete 
spaces are Polish. A subspace $A$ of a Polish space $X$ is Polish (with the relative topology) if and only if $A$ is $\mathbf{G}_{\delta}$ (i.e. a countable intersection of open sets) in $X$. The class of Polish spaces is closed under countable products; in particular, giving the discrete topology to $2=\{0,1\}$ and to $\mathbb{N}$, the Cantor space $\mathcal{C}=2^{\mathbb{N}}$ and the Baire space $\mathcal{N}=\mathbb{N}^{\mathbb{N}}$ are Polish. The former is homeomorphic to Cantor's $1 / 3$-set, the latter is homeomorphic to $\mathbb{R} \backslash \mathbb{Q}$.

For $X$ a topological Hausdorff space $\mathbf{F}(X)$ and $\mathbf{K}(X)$ are the collections of all closed, resp. compact, subsets of $X$. We can endow $\mathbf{F}(X)$ with a compact topology, the so-called Fell topology, which is generated by the sets of the form

$$
\left\{F \in \mathbf{F}(X) \mid F \cap K=\emptyset \& F \cap U_{1} \neq \emptyset \& \ldots \& F \cap U_{n} \neq \emptyset\right\},
$$

with $K \in \mathbf{K}(X)$ and $U_{1}, \ldots, U_{n}$ open in $X$. If $X$ is locally compact and second countable the Fell topology is Polish. If $d$ is a compatible metric on $X$ we can endow $\mathbf{F}(X) \backslash\{\emptyset\}$ with the Wijsman topology, i.e. the topology generated by the maps $\mathbf{F}(X) \backslash\{\emptyset\} \rightarrow \mathbb{R}, F \mapsto d(x, F)$, for $x \in X$. If $d$ is such that closed and bounded subsets are compact (e.g. if $X=\mathbb{R}^{n}$ with the usual metric), then the Wijsman topology coincides with the Fell topology restricted to $\mathbf{F}(X) \backslash\{\emptyset\}$. If $X$ is compact with compatible metric $d \leq 1$ the Fell topology on $\mathbf{F}(X)$ is the more familiar Vietoris topology on $\mathbf{K}(X)$, generated by the Hausdorff distance

$$
d^{\mathrm{H}}(K, L)=\max (\max \{d(x, K) \mid x \in L\}, \max \{d(x, L) \mid x \in K\}) .
$$

For more on the various topologies on $\mathbf{F}(X)$ see [2].

A basic way of classifying subsets of a Polish space (or even just of a topological space) $X$ according to their "smallness" is provided by Baire category. A subset of $X$ is nowhere dense if its closure has empty interior, meager (or of the first category) if it is the countable union of nowhere dense sets, non-meager (or of the second category) if it is not meager, comeager (or residual) if its complement with respect to $X$ is meager. The Baire category theorem asserts that no open set in a Polish space is meager or, equivalently, that the intersection of countably many dense open subsets of a Polish space is dense.

Most Polish spaces we will deal with will also have some natural algebraic structure compatible with the topology: mostly they are Fréchet spaces and hence Polish groups. A subgroup of a Polish group which is Polish is necessarily closed. Hence a $\mathbf{G}_{\delta}$ subset of a Polish group which is not closed is not a subgroup. Moreover, a non-meager Borel subgroup of a Polish group is Polish and hence a non-meager Borel subset of a Polish group which is not closed is not a subgroup.

1.2. The Borel hierarchy. For $X$ a Polish space (or even just a topological space) we can define the collection of Borel subsets of $X, \mathbf{B}(X)$. Although 
$\mathbf{B}(X)$ is a reasonable playground for most constructions in analysis, it lacks an important closure property - it is not closed under Borel or even continuous images. A subset $A$ of a Polish space $X$ is analytic if $A=f(B)$ for some Borel set $B$ in a Polish space $Y$ and some Borel function $f: Y \rightarrow X$. In fact, $f$ can be taken to be continuous and $B=Y$. Analytic sets are also called Suslin spaces or $\boldsymbol{\Sigma}_{1}^{1}$ sets; $\boldsymbol{\Sigma}_{1}^{1}(X)$ is the collection of all analytic subsets of $X$. Although $\boldsymbol{\Sigma}_{1}^{1}(X)$ contains $\mathbf{B}(X)$, and is closed under Borel images, countable intersections and countable unions, it is not closed under complements. The complement of an analytic set is called coanalytic or $\boldsymbol{\Pi}_{1}^{1}$ and we have the class $\boldsymbol{\Pi}_{1}^{1}(X)$ of all coanalytic subsets of $X$. Let us agree that $\boldsymbol{\Delta}_{1}^{1}(X)=\boldsymbol{\Sigma}_{1}^{1}(X) \cap \mathbf{\Pi}_{1}^{1}(X)$. A basic theorem of Lusin says that for $X$ a Polish space, $\mathbf{B}(X)=\boldsymbol{\Delta}_{1}^{1}(X)$.

The elements of $\mathbf{B}(X)$ are classified in a hierarchy. Let $\boldsymbol{\Sigma}_{1}^{0}(X)$ be the collection of open sets of $X$, let $\boldsymbol{\Pi}_{1}^{0}(X)$ be the collection of closed sets of $X$, and let $\boldsymbol{\Delta}_{1}^{0}(X)$ be the collection of clopen sets of $X$, i.e. $\boldsymbol{\Delta}_{1}^{0}(X)=$ $\boldsymbol{\Sigma}_{1}^{0}(X) \cap \boldsymbol{\Pi}_{1}^{0}(X)$. Next, let

$$
\begin{aligned}
& \boldsymbol{\Sigma}_{n+1}^{0}(X)=\left\{\bigcup_{i \in \mathbb{N}} A_{i} \mid A_{i} \in \boldsymbol{\Pi}_{n}^{0}(X)\right\}, \\
& \boldsymbol{\Pi}_{n+1}^{0}(X)=\left\{X \backslash A \mid A \in \boldsymbol{\Sigma}_{n+1}^{0}(X)\right\}, \\
& \boldsymbol{\Delta}_{n+1}^{0}(X)=\boldsymbol{\Sigma}_{n+1}^{0}(X) \cap \mathbf{\Pi}_{n+1}^{0}(X) .
\end{aligned}
$$

Thus $\boldsymbol{\Sigma}_{2}^{0}(X)$ is just $\mathbf{F}_{\sigma}(X)$ (i.e. the collection of countable unions of closed subsets of $X), \boldsymbol{\Pi}_{2}^{0}(X)$ is $\mathbf{G}_{\delta}(X), \boldsymbol{\Sigma}_{3}^{0}(X)$ is $\mathbf{G}_{\delta \sigma}(X), \boldsymbol{\Pi}_{3}^{0}(X)$ is $\mathbf{F}_{\sigma \delta}$, and so on. If $X$ is Polish then $\boldsymbol{\Delta}_{n}^{0}(X) \subseteq \boldsymbol{\Sigma}_{n}^{0}(X), \boldsymbol{\Pi}_{n}^{0}(X) \subseteq \boldsymbol{\Delta}_{n+1}^{0}(X)$. Moreover, if $X$ is uncountable the inclusions are proper. (If $X$ is countable then every subset is $\boldsymbol{\Sigma}_{2}^{0}$.) We should also point out that if $X$ is Polish and uncountable, then the classes $\boldsymbol{\Sigma}_{n}^{0}(X), \boldsymbol{\Pi}_{n}^{0}(X), \boldsymbol{\Delta}_{n}^{0}(X)$ do not exhaust $\mathbf{B}(X)$. In fact, $\boldsymbol{\Sigma}_{\alpha}^{0}(X)$, $\boldsymbol{\Pi}_{\alpha}^{0}(X), \boldsymbol{\Delta}_{\alpha}^{0}(X)$ can be defined for every countable ordinal $1 \leq \alpha<\omega_{1}$, so that these classes form a hierarchy and $\mathbf{B}(X)=\bigcup_{\alpha} \boldsymbol{\Sigma}_{\alpha}^{0}(X)=\bigcup_{\alpha} \boldsymbol{\Pi}_{\alpha}^{0}(X)=$ $\bigcup_{\alpha} \Delta_{\alpha}^{0}(X)$.

Now that we have defined this zoo of sets it is natural to seek a criterion to establish that, say, a certain Borel subset $A$ of a Polish space $X$ is a true $\boldsymbol{\Sigma}_{n}^{0}$ set, i.e. it is $\boldsymbol{\Sigma}_{n}^{0}$ but not $\boldsymbol{\Pi}_{n}^{0}$. In other words, we have to establish an upper bound for the complexity of $A$ and then show that no lower complexity is possible. Computing the upper bound in most (but not all!) cases is a fairly trivial matter and in any case can be achieved only by studying the original definition of the set at hand and/or some equivalent definition. We now turn to a powerful technique to establish lower bounds. Let $\boldsymbol{\Gamma}$ denote either $\boldsymbol{\Sigma}_{n}^{0}$, or $\boldsymbol{\Pi}_{n}^{0}$, or $\boldsymbol{\Sigma}_{1}^{1}$, or $\boldsymbol{\Pi}_{1}^{1}$. An easy inspection shows that $\boldsymbol{\Gamma}$ is closed under continuous preimages. We introduce the following terminology: for $A \subseteq X$, $B \subseteq Y$, with $X, Y$ Polish, we say that $B$ is Wadge reducible to $A$ (in symbols 
$\left.B \leq_{\mathrm{W}} A\right)$ if there is a continuous $f: Y \rightarrow X$ such that $B=f^{-1}(A)$. We say that $A \subseteq X$ is $\boldsymbol{\Gamma}$-hard if for every Polish space $Y$ and every $B \in \boldsymbol{\Gamma}(Y)$ we have $B \leq_{\mathrm{W}} A$. If $A$ is $\boldsymbol{\Gamma}$-hard then $X \backslash A$ cannot be in $\boldsymbol{\Gamma}$ and hence $\boldsymbol{\Gamma}$ is a lower bound for the complexity of $A$. If a $\Gamma$-hard set $A \subseteq X$ happens to be also in $\boldsymbol{\Gamma}(X)$ we say that $A$ is $\boldsymbol{\Gamma}$-complete. In this case we have pinned down the complexity of $A$.

A very useful technique for showing that a set $A \subseteq X$ (X Polish) is $\boldsymbol{\Gamma}$-hard is the following: choose a Polish space $Y$, a known $\boldsymbol{\Gamma}$-complete set $B \subseteq Y$ and show that $B \leq_{\mathrm{W}} A$. To apply this technique we need an array of examples of $\boldsymbol{\Gamma}$-complete sets; the following list provides some examples which will turn out to be useful in this paper. Details and proofs (together with more examples) can be found in Section 23 of [5]. Sections 27 and 33 of the same book contain many examples of $\boldsymbol{\Sigma}_{1}^{1}$-and $\boldsymbol{\Pi}_{1}^{1}$-complete sets.

EXAMPLES 1.1. $\forall^{\infty} n$ and $\exists^{\infty} n$ abbreviate respectively "for all but finitely many $n$ " and "for infinitely many $n$ ".

$$
\begin{aligned}
Q_{2} & =\left\{\alpha \in \mathcal{C} \mid \forall \forall^{\infty} n \alpha(n)=0\right\} & & \text { is } \boldsymbol{\Sigma}_{2}^{0} \text {-complete; } \\
N_{2} & =\left\{\alpha \in \mathcal{C} \mid \exists^{\infty} n \alpha(n)=0\right\} & & \text { is } \boldsymbol{\Pi}_{2}^{0} \text {-complete; } \\
S_{3} & =\left\{\alpha \in 2^{\mathbb{N} \times \mathbb{N}} \mid \exists n \exists^{\infty} m \alpha(n, m)=0\right\} & & \text { is } \boldsymbol{\Sigma}_{3}^{0} \text {-complete; } \\
P_{3} & =\left\{\alpha \in 2^{\mathbb{N} \times \mathbb{N}} \mid \forall n \forall^{\infty} m \alpha(n, m)=1\right\} & & \text { is } \boldsymbol{\Pi}_{3}^{0} \text {-complete; } \\
S_{4} & =\left\{\alpha \in 2^{\mathbb{N} \times \mathbb{N}} \mid \forall \forall^{\infty} n \forall^{\infty} m \alpha(n, m)=0\right\} & & \text { is } \boldsymbol{\Sigma}_{4}^{0} \text {-complete; } \\
\ell^{1} & =\left\{\left(x_{n}\right) \in[0,1]^{\mathbb{N}} \mid \sum x_{n}<+\infty\right\} & & \text { is } \boldsymbol{\Sigma}_{2}^{0} \text {-complete. }
\end{aligned}
$$

If we are dealing with $\mathbf{F}_{\sigma}$ 's or $\mathbf{G}_{\delta}$ 's we can also resort to the Baire category theorem to prove $\boldsymbol{\Sigma}_{2}^{0}$ or $\boldsymbol{\Pi}_{2}^{0}$-completeness. Indeed, the intersection of two dense $\mathbf{G}_{\delta}$ 's is non-empty and thus if $A$ and its complement $X \backslash A$ are both dense $A$ cannot be both $\mathbf{G}_{\delta}$ and $\mathbf{F}_{\sigma}$; establishing that one of the two possibilities holds in this case will therefore rule out the other. Notice also that an $\mathbf{F}_{\sigma}$ with dense complement is meager.

1.3. Sets of uniqueness. We will now review a few descriptive set-theoretic results that will be used in the sequel. The first theorem, in some sense, motivated much of the present paper. For a proof see e.g. [5], p. 123.

Theorem 1.2 (Lusin). Suppose $X$ and $Y$ are Polish spaces and $B \subseteq$ $X \times Y$ is Borel. Then $U=\{x \in X \mid \exists ! y(x, y) \in B\} \in \Pi_{1}^{1}(X)$.

This result gives an upper bound on the complexity of $U$. Sharper upper bounds can be obtained by putting restrictions on $B$. Let us call a space $\mathbf{K}_{\sigma}$ if it is a countable union of compact sets. An important theorem by Arsenin and Kunugui (see [5], p. 297) asserts that if for all $y \in Y$ the section $B_{y}=\{x \in X \mid(x, y) \in B\}$ is $\mathbf{K}_{\sigma}$, then $\operatorname{proj}_{X}(B)$ is Borel and hence $U$ is also Borel. 
We can gain even more control on the complexity of $\operatorname{proj}_{X}(B)$ and $U$ if we assume that $Y$ itself is $\mathbf{K}_{\sigma}$.

Lemma 1.3. Let $X$ be Polish and $Y$ metrizable and $\mathbf{K}_{\sigma}$. If $F \subseteq X \times Y$ is $\boldsymbol{\Sigma}_{2}^{0}$ then $\operatorname{proj}_{X}(F)$ is also $\boldsymbol{\Sigma}_{2}^{0}$.

Proof. First suppose $F$ is closed. Let $Y=\bigcup_{n} K_{n}$ with $K_{n}$ compact and let $F_{n}=F \cap\left(X \times K_{n}\right)$. As $\operatorname{proj}_{X}(F)=\bigcup_{n} \operatorname{proj}_{X}\left(F_{n}\right)$, it is enough to show that each $\operatorname{proj}_{X}\left(F_{n}\right)$ is closed in $X$. Let $x_{i} \in \operatorname{proj}_{X}\left(F_{n}\right)$ be such that $x_{i} \rightarrow x$. Pick $y_{i} \in K_{n}$ such that $\left(x_{i}, y_{i}\right) \in F_{n}$. By compactness of $K_{n}$ we can find a subsequence $\left(y_{i_{k}}\right)_{k}$ converging to some $y \in K_{n}$. By the closure of $F_{n}$ we have $\lim _{k \rightarrow \infty}\left(x_{i_{k}}, y_{i_{k}}\right)=(x, y) \in F_{n}$, so $x \in \operatorname{proj}_{X}\left(F_{n}\right)$. Thus $\operatorname{proj}_{X}\left(F_{n}\right)$ is closed.

The general case follows from the preceding case and from $\operatorname{proj}_{X}\left(\bigcup_{i} F_{i}\right)$ $=\bigcup_{i} \operatorname{proj}_{X}\left(F_{i}\right)$.

The result is false if we replace " $F$ is $\boldsymbol{\Sigma}_{2}^{0}$ " with " $F$ is $\boldsymbol{\Pi}_{2}^{0}$ ": every $\boldsymbol{\Sigma}_{1}^{1}$ subset of $X$ is the projection of a $\Pi_{2}^{0}$ subset of $X \times Y$, where $Y$ is the Cantor space.

Theorem 1.4. Let $X$ and $Y$ be Polish with $Y \mathbf{K}_{\sigma}$. Let $F \subseteq X \times Y$ be $\boldsymbol{\Sigma}_{2}^{0}$. Then $U=\{x \in X \mid \exists ! y \in Y(x, y) \in F\}$ is $D_{2}\left(\boldsymbol{\Sigma}_{2}^{0}\right)$, i.e. the difference of two $\boldsymbol{\Sigma}_{2}^{0}$ sets. Moreover, if $\operatorname{proj}_{X}(F)=X$, then $U$ is $\boldsymbol{\Pi}_{2}^{0}$.

Proof. Let

$$
M=\left\{x \in X \mid \exists y_{1}, y_{2} \in Y\left[y_{1} \neq y_{2} \&\left(x, y_{1}\right) \in F \&\left(x, y_{2}\right) \in F\right]\right\} .
$$

Then $M=\operatorname{proj}_{X}\left(\bigcup_{\varepsilon \in \mathbb{Q}^{+}} S_{\varepsilon}\right)$ where

$$
S_{\varepsilon}=\left\{\left(x, y_{1}, y_{2}\right) \in X \times Y^{2} \mid d\left(y_{1}, y_{2}\right) \geq \varepsilon \&\left(x, y_{1}\right) \in F \&\left(x, y_{2}\right) \in F\right\}
$$

and $d$ is a compatible metric on $Y$. $Y^{2}$ is $\mathbf{K}_{\sigma}$, being the product of two $\mathbf{K}_{\sigma}$ 's, and $S_{\varepsilon}$ is $\boldsymbol{\Sigma}_{2}^{0}$ in $X \times Y^{2}$. Therefore $\bigcup_{\varepsilon \in \mathbb{Q}^{+}} S_{\varepsilon}$ is $\boldsymbol{\Sigma}_{2}^{0}$. By Lemma 1.3 both $M$ and $\operatorname{proj}_{X}(F)$ are in $\Sigma_{2}^{0}(X)$. Hence $U=\operatorname{proj}_{X}(F) \backslash M \in D_{2}\left(\boldsymbol{\Sigma}_{2}^{0}\right)(X)$.

If $\operatorname{proj}_{X}(F)=X$ then $U=X \backslash M \in \Pi_{2}^{0}(X)$.

1.4. Functional Polish spaces. In order to study ordinary differential equations with the techniques of descriptive set theory we will need to consider spaces of functions defined on $\mathbb{R}^{2}$. These spaces are obviously interesting in their own right and in a forthcoming paper we will prove in detail and in greater generality some of the facts we just mention here.

For every $n$ let $K_{n}=[-n, n] \times[-n, n]$, so that every compact subset of $\mathbb{R}^{2}$ is contained in some $K_{n}$. We define a metric on $C\left(\mathbb{R}^{2}\right)$, the set of continuous functions from $\mathbb{R}^{2}$ to $\mathbb{R}$, by setting, for each $F, G \in C\left(\mathbb{R}^{2}\right)$ and $n \in \mathbb{N}$,

$$
d_{n}(F, G)=\frac{\|(F-G)\left\lceil K_{n} \|_{\infty}\right.}{1+\|(F-G)\left\lceil K_{n} \|_{\infty}\right.},
$$


so that $d_{n}(F, G)<1$, and then defining

$$
d(F, G)=\sum_{n=1}^{\infty} \frac{d_{n}(F, G)}{2^{n}} .
$$

This metric induces the compact-open topology: $\lim _{k \rightarrow \infty} F_{k}=F$ if and only if $F_{k}$ converges to $F$ uniformly on each $K_{n}$ and hence on every compact subset of $X$. It is straightforward to check that $C\left(\mathbb{R}^{2}\right)$ equipped with this metric is a Polish space.

For various purposes different subspaces of $C\left(\mathbb{R}^{2}\right)$ will turn out to be useful; in general, they are not Polish with the topology they inherit from $C\left(\mathbb{R}^{2}\right)$, but some of them are Polish with appropriate topologies which exploit their peculiarities.

For example, let $C_{00}\left(\mathbb{R}^{2}\right)=\left\{F \in C\left(\mathbb{R}^{2}\right) \mid F\right.$ has compact support $\}$ equipped with the sup metric and let $C_{0}\left(\mathbb{R}^{2}\right)$ be the completion of $C_{00}\left(\mathbb{R}^{2}\right)$. $C_{0}\left(\mathbb{R}^{2}\right)$ is Polish (with the sup metric as a complete compatible metric) and its elements are the functions vanishing at infinity.

$C_{00}\left(\mathbb{R}^{2}\right)$ is $\boldsymbol{\Sigma}_{2}^{0}$-complete both as a subset of $C\left(\mathbb{R}^{2}\right)$ and as a subset of $C_{0}\left(\mathbb{R}^{2}\right)$, while $C_{0}\left(\mathbb{R}^{2}\right)$ is $\Pi_{3}^{0}$-complete as a subset of $C\left(\mathbb{R}^{2}\right)$.

A generalization of $C_{0}\left(\mathbb{R}^{2}\right)$ is given by the following definition.

Definition 1.5. Let $C_{\mathrm{c}}\left(\mathbb{R}^{2}\right)$ be the set of all $F \in C\left(\mathbb{R}^{2}\right)$ such that there exists $L \in \mathbb{R}$ such that for every $\varepsilon>0$ there exists $n$ such that for every $(t, u) \in \mathbb{R}^{2} \backslash K_{n}$ we have $|F(t, u)-L|<\varepsilon$. The elements of $C_{\mathrm{c}}\left(\mathbb{R}^{2}\right)$ are called functions constant at infinity.

The sup metric can be used to turn $C_{\mathrm{c}}\left(\mathbb{R}^{2}\right)$ into a Polish space (homeomorphic to $C_{0}\left(\mathbb{R}^{2}\right) \times \mathbb{R}$ via the obvious bijection). $C_{\mathrm{c}}\left(\mathbb{R}^{2}\right)$ has also another natural topology, namely the one inherited from $C\left(\mathbb{R}^{2}\right)$, which is not Polish because $C_{\mathrm{c}}\left(\mathbb{R}^{2}\right)$ is $\Pi_{3}^{0}$-complete as a subset of $C\left(\mathbb{R}^{2}\right)$.

Another interesting subset of $C\left(\mathbb{R}^{2}\right)$ consists of the functions satisfying the Lipschitz condition. This set is particularly interesting when studying functions that appear in ordinary differential equations because the Lipschitz condition is the best known condition that insures the uniqueness of the solution of these equations. With this in mind we will limit ourselves to the Lipschitz condition in the second variable.

Definition 1.6. Let $\mathrm{Lip}_{2}$ be the set of functions in $C\left(\mathbb{R}^{2}\right)$ such that there exists $L$ such that for all $t, u_{0}, u_{1} \in \mathbb{R}$ we have $\left|F\left(t, u_{0}\right)-F\left(t, u_{1}\right)\right|<$ $L\left|u_{0}-u_{1}\right|$.

$\mathrm{Lip}_{2}$ is $\boldsymbol{\Sigma}_{2}^{0}$-complete as a subset of $C\left(\mathbb{R}^{2}\right)$. Moreover, $C_{00}\left(\mathbb{R}^{2}\right), C_{0}\left(\mathbb{R}^{2}\right)$, $C_{\mathrm{c}}\left(\mathbb{R}^{2}\right)$, and $\mathrm{Lip}_{2}$ are all meager in $C\left(\mathbb{R}^{2}\right)$. 
2. Generalities on ordinary differential equations. In this section we begin the study of ordinary differential equations. A Cauchy problem is a triple $(F, x, y) \in C\left(\mathbb{R}^{2}\right) \times \mathbb{R}^{2}$ which gives the initial value problem

$$
\varphi^{\prime}(t)=\frac{d \varphi}{d t}=F(t, \varphi(t)), \quad \varphi(x)=y .
$$

The Cauchy/Peano theorem asserts that this problem always has a local solution (i.e. a solution $\varphi$ defined in some neighborhood of $x$ ). Moreover, if $F$ is bounded (and hence, in particular, if it belongs to $C_{\mathrm{c}}\left(\mathbb{R}^{2}\right)$, which has the advantage of being Polish with the sup metric) the Cauchy problem always has a global solution, i.e. a solution defined on the whole real line.

We first consider Cauchy problems with $F \in C_{\mathrm{c}}\left(\mathbb{R}^{2}\right)$. The solutions of this kind of ordinary differential equations are continuously differentiable functions, i.e. elements of $C^{1}(\mathbb{R})$; this is obviously a subspace of $C(\mathbb{R})$ (endowed with the compact-open topology) and is $\boldsymbol{\Pi}_{3}^{0}$-complete (see [5], p. 183). We could easily give $C^{1}(\mathbb{R})$ a Polish topology, but this will not be needed.

Definition 2.1. Let $\mathcal{S}^{\mathrm{c}}$ be the set of all $(F, x, y, \varphi) \in C_{\mathrm{c}}\left(\mathbb{R}^{2}\right) \times \mathbb{R}^{2} \times C(\mathbb{R})$ such that

$$
\varphi \in C^{1}(\mathbb{R}) \& \forall t \in \mathbb{R}\left[\varphi^{\prime}(t)=F(t, \varphi(t))\right] \& \varphi(x)=y .
$$

If $F \in C_{\mathrm{c}}\left(\mathbb{R}^{2}\right)$ and $(x, y) \in \mathbb{R}^{2}$ let

$$
\mathcal{S}_{F,(x, y)}^{\mathrm{c}}=\left\{\varphi \in C(\mathbb{R}) \mid(F, x, y, \varphi) \in \mathcal{S}^{\mathrm{c}}\right\} .
$$

In other words, $\mathcal{S}_{F,(x, y)}^{\mathrm{c}}$ is the set of all solutions of the Cauchy problem given by $(F, x, y)$.

The Cauchy/Peano theorem implies that for every $F \in C_{\mathrm{c}}\left(\mathbb{R}^{2}\right)$ and $(x, y) \in \mathbb{R}^{2}$ we have $\mathcal{S}_{F,(x, y)}^{\mathrm{c}} \neq \emptyset$. In general, there is no uniqueness of the solution of the Cauchy problem, and the classical counterexample (which will be exploited in what follows) is given by any function $F \in C_{\mathrm{c}}\left(\mathbb{R}^{2}\right)$ such that in a neighborhood of $(0,0)$ we have $F(t, u)=\sqrt{|u|}$. For such an $F, \mathcal{S}_{F,(0,0)}^{\mathrm{c}}$ includes functions $\varphi$ that locally satisfy either $\varphi(t)=0$ or $\varphi(t)=\frac{1}{4} t^{2}$. In general, if $\mathcal{S}_{F,(x, y)}^{\mathrm{c}}$ is not a singleton then it has the cardinality of the continuum.

The following lemma is our version of the so-called continuous dependence from parameters of solutions of ODEs.

LEMMA 2.2. $\mathcal{S}^{\mathrm{c}}$ is closed.

Proof. Suppose $\left\{\left(F_{n}, x_{n}, y_{n}, \varphi_{n}\right)\right\}_{n}$ is a sequence of elements of $\mathcal{S}^{\mathrm{c}}$ and

$$
\lim _{n \rightarrow \infty}\left(F_{n}, x_{n}, y_{n}, \varphi_{n}\right)=(F, x, y, \varphi) .
$$

To check that $\varphi$ satisfies $\varphi^{\prime}(t)=F(t, \varphi(t))$ fix $t$ and work in the interval $I_{t}=[t-1, t+1]$. The $\varphi_{n}$ 's are all $C^{1}$ on $I_{t}$ and their derivatives, being 
$F_{n}\left(t, \varphi_{n}(t)\right)$, converge uniformly on $I_{t}$. Therefore $\varphi$ is also $C^{1}$ on $I_{t}$ and $\lim _{n \rightarrow \infty} \varphi_{n}^{\prime}=\varphi^{\prime}$. Given $\varepsilon>0$, since $F$ is uniformly continuous on $Q_{t}=$ $I_{t} \times[-K-1, K+1]$, where $K=\left\|\varphi \mid I_{t}\right\|_{\infty}$, there exists $\delta>0$ such that $|b-c|<\delta \Rightarrow|F(a, b)-F(a, c)|<\varepsilon / 2$ for every $(a, b),(a, c) \in Q_{t}$. If $n$ is sufficiently large we have both $\left\|F_{n}-F\right\|_{\infty}<\varepsilon / 2$ and $\|\left(\varphi_{n}-\varphi\right)\left\lceil I_{t} \|_{\infty}<\delta\right.$. For such an $n$, the triangle inequality implies that $\left|F_{n}\left(t, \varphi_{n}(t)\right)-F(t, \varphi(t))\right|<\varepsilon$, for every $t \in I_{t}$. This yields

$$
\varphi^{\prime}(t)=\lim _{n \rightarrow \infty} \varphi_{n}^{\prime}(t)=\lim _{n \rightarrow \infty} F_{n}\left(t, \varphi_{n}(t)\right)=F(t, \varphi(t)) .
$$

To show that $\varphi(x)=y$, observe that $\varphi$ is uniformly continuous and that on some neighborhood of $x$ the $\varphi_{n}$ 's converge uniformly to $\varphi$. Hence for every $\varepsilon>0$ if $n$ is sufficiently large we see that $\left|y_{n}-y\right|,\left|\varphi_{n}\left(x_{n}\right)-\varphi\left(x_{n}\right)\right|$, and $\left|\varphi\left(x_{n}\right)-\varphi(x)\right|$ are all smaller than $\varepsilon / 3$. Using the triangle inequality and $\varphi_{n}\left(x_{n}\right)=y_{n}$ this yields $|\varphi(x)-y|<\varepsilon$ and completes the proof that $(F, x, y, \varphi) \in \mathcal{S}^{\mathrm{c}}$.

It is not true that for every $F \in C\left(\mathbb{R}^{2}\right)$ and $(x, y) \in \mathbb{R}^{2}$ there exists a global solution of the Cauchy problem given by $(F, x, y)$. In fact, in this case the Cauchy/Peano theorem asserts only the existence of a local solution and the classical counterexample is $F(t, u)=u^{2}$, which admits the global solution $\varphi(t)=0$ only when $y=0$, while for other initial conditions it has non-global solutions of the form $\varphi(t)=-(t+c)^{-1}$.

A solution of a Cauchy problem is called non-extendible if its domain is connected and there is no solution properly extending it and having connected domain. An immediate consequence of the Cauchy/Peano theorem is that the domain of a non-extendible solution is an open interval. The continuity of $F$ implies that if $a \in \mathbb{R}$ is a limit of the domain of the non-extendible solution $\varphi$, then the vertical line $t=a$ is an asymptote for the graph of $\varphi$. This entails that the graph of any non-extendible solution is a closed subset of $\mathbb{R}^{2}$, i.e. an element of $\mathbf{F}\left(\mathbb{R}^{2}\right)$. Hence we can look for solutions of a Cauchy problem in $\mathbf{F}\left(\mathbb{R}^{2}\right)$ endowed with the Fell topology.

Definition 2.3. Let $C_{\mathrm{par}}^{1}(\mathbb{R})$ be the set of all $C^{1}$ functions $\varphi$ defined on an open non-empty interval $(a, b)$ such that if $a>-\infty$ then $\lim _{t \rightarrow a+} \varphi(t)=$ $\pm \infty$ and if $b<+\infty$ then $\lim _{t \rightarrow b-} \varphi(t)= \pm \infty . C_{\mathrm{par}}^{1}(\mathbb{R})$ is endowed with the topology obtained by identifying $\varphi$ with its graph in $\mathbf{F}\left(\mathbb{R}^{2}\right)$.

The "par" in $C_{\mathrm{par}}^{1}(\mathbb{R})$ stands for "partial".

Definition 2.4. Let $\mathcal{S}$ be the set of all $(F, x, y, \varphi) \in C\left(\mathbb{R}^{2}\right) \times \mathbb{R}^{2} \times C_{\mathrm{par}}^{1}(\mathbb{R})$ such that

$$
\forall t \in \operatorname{dom}(\varphi)\left[\varphi^{\prime}(t)=F(t, \varphi(t))\right] \& x \in \operatorname{dom}(\varphi) \& \varphi(x)=y .
$$


If $F \in C\left(\mathbb{R}^{2}\right)$ and $(x, y) \in \mathbb{R}^{2}$ let

$$
\mathcal{S}_{F,(x, y)}=\left\{\varphi \in C_{\mathrm{par}}^{1}(\mathbb{R}) \mid(F, x, y, \varphi) \in \mathcal{S}\right\} .
$$

In other words, $\mathcal{S}_{F,(x, y)}$ is the set of all non-extendible solutions of the Cauchy problem $(F, x, y)$.

The following lemma will turn out to be useful in studying $\mathcal{S}$.

Lemma 2.5. Let $\left\{\left(F_{n}, x_{n}, y_{n}\right)\right\}_{n}$ be a sequence of elements of $C\left(\mathbb{R}^{2}\right) \times \mathbb{R}^{2}$ which converges to $(F, x, y)$. For every $n$ let $\varphi_{n} \in \mathcal{S}_{F_{n},\left(x_{n}, y_{n}\right)}$. Then for all but finitely many $n$ we have $x \in \operatorname{dom}\left(\varphi_{n}\right)$. Moreover, $\lim _{n \rightarrow \infty} \varphi_{n}(x)=y$.

Proof. Suppose, towards a contradiction, that for infinitely many $n$, $x \notin \operatorname{dom}\left(\varphi_{n}\right)$; we can extract a subsequence so that $x \notin \operatorname{dom}\left(\varphi_{n}\right)$ for every $n$. Since either infinitely often $x_{n}>x$ or infinitely often $x_{n}<x$ we can suppose the first possibility holds (the other can be dealt with symmetrically); by extracting a subsequence we can assume that for every $n, x_{n}>x$. This implies that there exists $a_{n}$ such that $x \leq a_{n}<x_{n}$ and $t=a_{n}$ is a vertical asymptote for $\varphi_{n}$. By refining our sequence we may assume that for every $n$,

$$
\lim _{t \rightarrow a_{n}+} \varphi_{n}(t)=-\infty \quad \text { and } \quad x_{n}-x \leq 2^{-2 n} .
$$

(The case $\lim _{t \rightarrow a_{n}+} \varphi_{n}(t)=+\infty$ is analogous.)

Let $z_{n}$ be such that $a_{n}<z_{n}<x_{n}, \varphi_{n}\left(z_{n}\right)=y_{n}-2^{-n}$, and $\varphi_{n}(t)>$ $y_{n}-2^{-n}$ if $z_{n}<t \leq x_{n}$. Let $u_{n}$ be such that $z_{n}<u_{n} \leq x_{n}, y_{n} \leq \varphi_{n}\left(u_{n}\right) \leq$ $y_{n}+2^{-n}$, and $\varphi_{n}(t)<y_{n}+2^{-n}$ if $z_{n}<t<u_{n}$. Since $\varphi_{n}$ is $C^{1}$, by the Mean Value Theorem, there exists $w_{n}$ such that $z_{n} \leq w_{n} \leq u_{n}$ and

$$
\varphi_{n}^{\prime}\left(w_{n}\right)=\frac{\varphi_{n}\left(u_{n}\right)-\varphi_{n}\left(z_{n}\right)}{u_{n}-z_{n}} \geq \frac{y_{n}-\left(y_{n}-2^{-n}\right)}{x_{n}-x} \geq \frac{2^{-n}}{2^{-2 n}}=2^{n}
$$

(we used $x \leq a_{n} \leq z_{n}<u_{n} \leq x_{n}$ and $x_{n}-x \leq 2^{-2 n}$ ). Hence (using the fact that $\left.\varphi_{n} \in \mathcal{S}_{F_{n},\left(x_{n}, y_{n}\right)}\right)$ we have

$$
F_{n}\left(w_{n}, \varphi_{n}\left(w_{n}\right)\right)=\varphi_{n}^{\prime}\left(w_{n}\right) \geq 2^{n} .
$$

Therefore $\lim _{n \rightarrow \infty} F_{n}\left(w_{n}, \varphi_{n}\left(w_{n}\right)\right)=+\infty$.

Since $\left|y_{n}-\varphi_{n}\left(w_{n}\right)\right| \leq 2^{-n}$ and $x<w_{n} \leq x_{n}$, we have $\left\{\left(w_{n}, \varphi_{n}\left(w_{n}\right)\right)\right\}_{n}$ $\rightarrow(x, y)$. This and $F_{n} \rightarrow F$ imply that $\lim _{n \rightarrow \infty} F_{n}\left(w_{n}, \varphi_{n}\left(w_{n}\right)\right)=F(x, y)$, contradicting what was shown above, and proving the first part of the lemma.

We can now suppose that $\varphi_{n}(x)$ is defined for every $n$ and prove the second part of the lemma similarly. Again we suppose that $x_{n}>x$ for every $n$. Assume towards a contradiction that the sequence $\left\{\varphi_{n}(x)\right\}_{n}$ does not converge to $y$. Then for some subsequence and some $\varepsilon>0$ we can assume that for all $n, \varphi_{n}(x) \leq y-\varepsilon$ (or the symmetric case with $\geq$ and + ) and $y_{n}>y-\varepsilon / 2$. Let $z_{n}$ be such that $\varphi_{n}\left(z_{n}\right)=y-\varepsilon$ and $\varphi_{n}(t)>y-\varepsilon$ if 
$z_{n}<t \leq x_{n}$. As above (using the appropriate $u_{n}$ ) there exists $w_{n}$ such that $\varphi_{n}\left(w_{n}\right) \leq y$ and

$$
F_{n}\left(w_{n}, \varphi_{n}\left(w_{n}\right)\right)=\varphi_{n}^{\prime}\left(w_{n}\right) \geq \frac{y_{n}-\varphi_{n}\left(z_{n}\right)}{x_{n}-z_{n}} \geq \frac{\varepsilon}{2\left(x_{n}-x\right)} .
$$

Therefore $\lim _{n \rightarrow \infty} F_{n}\left(w_{n}, \varphi_{n}\left(w_{n}\right)\right)=+\infty$ holds also in this case.

Obviously, $w_{n} \rightarrow x$, but this time it may not be the case that $\varphi_{n}\left(w_{n}\right) \rightarrow$ $y$. We can reach a contradiction anyway by noticing that $y-\varepsilon \leq \varphi_{n}\left(w_{n}\right) \leq y$ and hence the sequence $\left\{\varphi_{n}\left(w_{n}\right)\right\}_{n}$ has an accumulation point.

Lemma 2.6. $\mathcal{S}$ is closed in $C\left(\mathbb{R}^{2}\right) \times \mathbb{R}^{2} \times C_{\text {par }}^{1}(\mathbb{R})$.

Pr o of. Suppose $\left\{\left(F_{n}, x_{n}, y_{n}, \varphi_{n}\right)\right\}_{n}$ is a sequence of elements of $\mathcal{S}$ such that

$$
\lim _{n \rightarrow \infty}\left(F_{n}, x_{n}, y_{n}, \varphi_{n}\right)=(F, x, y, \varphi)
$$

within $C\left(\mathbb{R}^{2}\right) \times \mathbb{R}^{2} \times C_{\mathrm{par}}^{1}(\mathbb{R})$. By Lemma 2.5 we may assume that $x \in$ $\operatorname{dom}\left(\varphi_{n}\right)$ for every $n$ and that $\lim _{n \rightarrow \infty} \varphi_{n}(x)=y$. Denote by $\Gamma$ the graph of $\varphi$ and by $\Gamma_{n}$ the graph of $\varphi_{n}$; these are elements of $\mathbf{F}\left(\mathbb{R}^{2}\right)$.

If $t \in \operatorname{dom}(\varphi)$ then for every $\varepsilon>0$ we see that $\Gamma$ intersects the open ball of center $(t, \varphi(t))$ and radius $\varepsilon$. Since $\Gamma_{n} \rightarrow \Gamma$ in $\mathbf{F}\left(\mathbb{R}^{2}\right)$, for all sufficiently large $n$ 's, $\Gamma_{n}$, has non-empty intersection with the same open ball, i.e. there exists $t_{n} \in \operatorname{dom}\left(\varphi_{n}\right)$ such that the distance between $\left(t_{n}, \varphi_{n}\left(t_{n}\right)\right)$ and $(t, \varphi(t))$ is less than $\varepsilon$. In other words, $\lim _{n \rightarrow \infty}\left(t_{n}, \varphi_{n}\left(t_{n}\right)\right)=(t, \varphi(t))$. By Lemma 2.5 this entails that for all but finitely many $n$ 's, $t \in \operatorname{dom}\left(\varphi_{n}\right)$ and also that $\lim _{n \rightarrow \infty} \varphi_{n}(t)=\varphi(t)$.

In particular, when $t=x$ we have $\varphi(x)=\lim _{n \rightarrow \infty} \varphi_{n}(x)=y$ and hence $\varphi$ satisfies the initial condition $(x, y)$.

Now we need to check that $\varphi^{\prime}(t)=F(t, \varphi(t))$ for every $t \in \operatorname{dom}(\varphi)$. To this end it suffices to restrict ourselves to some compact set and repeat the argument used in the proof of Lemma 2.2. Let $\varepsilon>0$ be such that $I_{t}=[t-\varepsilon, t+\varepsilon] \subset \operatorname{dom}(\varphi)$ and let $Q_{t}=\left\{(r, u)\left|r \in I_{t} \&\right| \varphi(r)-u \mid \leq 1\right\}$. $Q_{t}$ is compact and for all but finitely many $n$ 's the graph of $\varphi_{n}$ restricted to $I_{t}$ is contained in $Q_{t}$.

The previous lemma is not entirely satisfactory because $C\left(\mathbb{R}^{2}\right) \times \mathbb{R}^{2} \times$ $C_{\mathrm{par}}^{1}(\mathbb{R})$ is not a Polish space. By identifying a function with its graph, $\mathcal{S}$ can be also viewed as a subset of the Polish space $C\left(\mathbb{R}^{2}\right) \times \mathbb{R}^{2} \times \mathbf{F}\left(\mathbb{R}^{2}\right)$; in this space, $\mathcal{S}$ is not closed, but the elements of its closure which do not belong to it are close to being elements of $\mathcal{S}$, not only topologically but also from the point of view of the corresponding ODE. Indeed, we are now going to show that if $(F, x, y, \Gamma)$ belongs to the closure of $\mathcal{S}$ but not to $\mathcal{S}$ then $\Gamma$ is the graph of a $C^{1}$ function whose domain is not connected, but which is 
otherwise a non-extendible solution of the Cauchy problem given by $F$ and $(x, y)$.

LEMMA 2.7. Let $\left\{\left(F_{n}, x_{n}, y_{n}, \varphi_{n}\right)\right\}_{n}$ be a sequence of elements of $\mathcal{S}$ and let $\Gamma_{n}$ be the graph of $\varphi_{n}$. If

$$
\lim _{n \rightarrow \infty}\left(F_{n}, x_{n}, y_{n}, \Gamma_{n}\right)=(F, x, y, \Gamma)
$$

within $C\left(\mathbb{R}^{2}\right) \times \mathbb{R}^{2} \times \mathbf{F}\left(\mathbb{R}^{2}\right)$ then $(x, y) \in \Gamma, \Gamma$ is the graph of a function $\varphi$, and on each connected component of its domain $\varphi$ is a non-extendible solution of the $O D E \varphi^{\prime}(t)=F(t, \varphi(t))$.

Proof. First of all notice that $(x, y) \in \Gamma$ follows immediately from the fact that $\left(x_{n}, y_{n}\right) \in \Gamma_{n}$ for each $n$.

Then we need to show that $\Gamma$ is the graph of a function, i.e. that for each $t$ there exists at most one $u$ such that $(t, u) \in \Gamma$. Let $u^{1}$ and $u^{2}$ be such that $\left(t, u^{1}\right),\left(t, u^{2}\right) \in \Gamma$. Since $\Gamma_{n} \rightarrow \Gamma$ in $\mathbf{F}\left(\mathbb{R}^{2}\right)$, we may assume that for every $n$ there exist $t_{n}^{1}$ and $t_{n}^{2}$ such that the distance between $\left(t_{n}^{i}, \varphi_{n}\left(t_{n}^{i}\right)\right)$ and $\left(t, u^{i}\right)$ is less than $2^{-n}$. Hence $\left(F_{n}, t_{n}^{i}, \varphi_{n}\left(t_{n}^{i}\right)\right)$ converges to $\left(F, t, u^{i}\right)$. Lemma 2.5 implies $\lim _{n \rightarrow \infty} \varphi_{n}(t)=u^{i}$, which entails $u^{1}=u^{2}$.

Let $\varphi$ be the function with graph $\Gamma$. The continuity of $\varphi$ can be established by an argument similar to the one used in the preceding paragraph. To prove that $\varphi$ is $C^{1}$ and satisfies $\varphi^{\prime}(t)=F(t, \varphi(t))$ for every $t \in \operatorname{dom}(\varphi)$ we can argue exactly as in the proof of Lemma 2.2 (indeed, the arguments there were of local character).

To prove that on each connected component of its domain $\varphi$ is a nonextendible solution it suffices to show that these connected components are indeed open, since it will then follow from the closedness of $\Gamma$ that at the endpoints we have a vertical asymptote. Let $t \in \operatorname{dom}(\varphi)$. By Lemma 2.5 for all but finitely many $n$ we have $t \in \operatorname{dom}\left(\varphi_{n}\right)$ and $\lim _{n \rightarrow \infty} \varphi_{n}(t)=\varphi(t)$. For every $n$ such that $t \in \operatorname{dom}\left(\varphi_{n}\right)$ let $\varepsilon_{n}$ be maximal such that $\left(t-\varepsilon_{n}, t+\varepsilon_{n}\right) \subseteq$ $\operatorname{dom}\left(\varphi_{n}\right)$. If $\varepsilon=\liminf \varepsilon_{n}$ then clearly $(t-\varepsilon, t+\varepsilon) \subseteq \operatorname{dom}(\varphi)$ and therefore it suffices to show that $\varepsilon>0$. If $\varepsilon=0$, for the sake of simplicity suppose

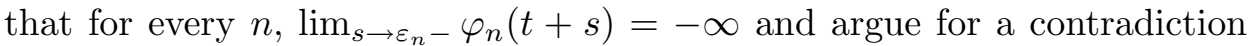
using the continuity of $F$, as in the proof of Lemma 2.5.

The following lemma will be the basic tool for showing the Wadge reducibility of certain sets of sequences to sets of Cauchy problems.

Lemma 2.8. Let $x_{0}, x_{1}, y_{0}, y_{1} \in \mathbb{R}$ with $x_{0}<x_{1}$ and $y_{0}<y_{1}$ and let $M>0$. There exists a continuous map $\mathcal{C} \rightarrow C\left(\left[x_{0}, x_{1}\right] \times\left[y_{0}, y_{1}\right]\right), \beta \mapsto G_{\beta}$, such that:

(1) $G_{\beta}(t, u)=0$ whenever $(t, u)$ belongs to the boundary of the rectangle $\left[x_{0}, x_{1}\right] \times\left[y_{0}, y_{1}\right]$

(2) $0 \leq G_{\beta}(t, u) \leq M$ for every $(t, u) \in\left[x_{0}, x_{1}\right] \times\left[y_{0}, y_{1}\right]$; 
(3) the constant function $\varphi(t)=y_{0}$ is a solution to $\left(G_{\beta}, x_{0}, y_{0}\right)$ on the interval $\left[x_{0}, x_{1}\right]$; any other solution $\varphi$ of this Cauchy problem on the right of $x_{0}$ is defined on the whole interval $\left[x_{0}, x_{1}\right]$ and satisfies $\varphi\left(x_{1}\right)>y_{0}$;

(4) $\left(G_{\beta}, x_{0}, y_{0}\right)$ has a unique solution if and only if $\beta \in N_{2}$ (see 1.1).

Proof. It suffices to prove the result for $x_{0}=y_{0}=0$ and $x_{1}=y_{1}=$ $M=1$, since an easy rescaling leads to the general result.

Let $g:(0,1] \rightarrow \mathbb{R}$ be a $C^{1}$ function such that:

(a) $g(t) \geq(\sqrt{t})^{-1}$ and $g\left(2^{-n}\right)=\sqrt{2^{n}}$;

(b) $g^{\prime}\left(2^{-n}\right)=-\frac{1}{2} \sqrt{2^{3 n}}$;

(c) $\int_{2^{-n-1}}^{2^{-n}} g(t) d t=1$.

For every $\beta \in \mathcal{C}$ we define $f_{\beta} \in C([0,1])$ by

$$
f_{\beta}(t)= \begin{cases}0 & \text { if } t=0, \\ \sqrt{t} & \text { if } 2^{-(n+1)}<t \leq 2^{-n} \text { and } \beta(n)=1, \\ 1 / g(t) & \text { if } 2^{-(n+1)}<t \leq 2^{-n} \text { and } \beta(n)=0\end{cases}
$$

so that $0 \leq f_{\beta}(t) \leq \sqrt{t}$ for every $t \in[0,1]$. By condition (a) we see that for every $\beta \in \mathcal{C}, f_{\beta}$ is continuous and that the map $\mathcal{C} \rightarrow C([0,1]), \beta \mapsto f_{\beta}$, is continuous. By condition (b), $f_{\beta}$ is $C^{1}$ on the interval $(0,1]$. Define $G_{\beta} \in$ $C\left([0,1]^{2}\right)$ by setting $G_{\beta}(t, u)=f_{\beta}(u)$. The map $\mathcal{C} \rightarrow C\left([0,1]^{2}\right), \beta \mapsto G_{\beta}$ is continuous and condition (2) holds.

(3) holds because $\varphi(t)=0$ is a solution of the differential equation for every $\beta \in \mathcal{C}$. If $\varphi$ is a non-zero solution then, since $0 \leq G_{\beta} \leq 1, \varphi$ is nondecreasing and satisfies $\varphi(t) \leq t$. Hence $\varphi$ is defined on $[0,1]$ and $\varphi(1)>0$ holds.

To check that (4) holds as well, notice that as $\int_{0}^{1} d t / \sqrt{t}$ converges, condition (c) above implies that for every $\beta \in \mathcal{C}$,

$$
\begin{aligned}
\int_{0}^{1} \frac{d t}{f_{\beta}(t)}<+\infty & \Leftrightarrow \sum_{n} \int_{2^{-n-1}}^{2^{-n}} \frac{d t}{f_{\beta}(t)}<+\infty \\
& \Leftrightarrow \sum_{n, \beta(n)=12^{-n-1}} \int_{n, \beta(n)=0}^{2^{-n}} \frac{d t}{\sqrt{t}}+\int_{2^{-n-1}}^{2^{-n}} g(t) d t<+\infty \\
& \Leftrightarrow \forall^{\infty} \beta(n)=1 .
\end{aligned}
$$

Therefore

$$
\int_{0}^{1} \frac{d t}{f_{\beta}(t)}=+\infty \Leftrightarrow \beta \in N_{2} .
$$

Thus to establish the first part of the lemma it suffices to show that the non-uniqueness of the solution of $\varphi^{\prime}(t)=G_{\beta}(t, \varphi(t))$ with initial condition $\varphi(0)=0$ is equivalent to the convergence of the integral $\int_{0}^{1} d t / f_{\beta}(t)$. 
Notice that if the equation has solutions different from $\varphi(t)=0$ then, since $G_{\beta}$ does not depend on the first variable and is positive, there exists at least one solution such that $\varphi(t)>0$ whenever $t>0$. For this $\varphi$ we have $\varphi^{\prime}(t) / f_{\beta}(\varphi(t))=1$ if $t>0$ and hence

$$
\int_{0}^{\varphi(1)} \frac{d t}{f_{\beta}(t)}=\int_{0}^{1} \frac{\varphi^{\prime}(s)}{f_{\beta}(\varphi(s))} d s=1,
$$

which shows that $\int_{0}^{1} d t / f_{\beta}(t)$ converges. For the other direction suppose $\int_{0}^{1} d t / f_{\beta}(t)$ converges and let $h(y)=\int_{0}^{y} d t / f_{\beta}(t)$; this is a strictly increasing function and hence it has an inverse $\varphi(t)$ which is also strictly increasing and satisfies $\varphi(0)=0$. Moreover, $\varphi^{\prime}(t)=f_{\beta}(\varphi(t))=G_{\beta}(t, \varphi(t))$ and hence $\varphi$ is a non-zero solution of the differential equation.

To verify (1) notice that our construction only ensures $G_{\beta}=0$ on the lower side of the square. If we apply this construction to the square $[1 / 3,2 / 3] \times[0,1 / 3]$ we can use the area between the two squares as a "buffer zone" where $G_{\beta}$ is defined to be Lipschitz in the second variable, so that (4) still holds. This can easily be done preserving the continuity both of $G_{\beta}$ itself and of the map $\beta \mapsto G_{\beta}$.

3. Cauchy problems with a unique solution. Given a Cauchy problem $(F, x, y)$ we say that $(\bar{x}, \bar{y})$ is a bifurcation point if and only if for every $\varepsilon>0$ there exist two solutions to $(F, x, y)$ going through $(\bar{x}, \bar{y})$ which differ at some point of $(\bar{x}-\varepsilon, \bar{x}+\varepsilon)$.

3.1. Uniqueness in $C_{\mathrm{c}}\left(\mathbb{R}^{2}\right)$.

DeFinition 3.1. Let

$$
\begin{aligned}
\mathcal{U}^{\mathrm{c}} & =\left\{(F, x, y) \in C_{\mathrm{c}}\left(\mathbb{R}^{2}\right) \times \mathbb{R}^{2} \mid \exists ! \varphi\left(\varphi \in \mathcal{S}_{F,(x, y)}^{\mathrm{c}}\right)\right\}, \\
\mathcal{M}^{\mathrm{c}} & =C_{\mathrm{c}}\left(\mathbb{R}^{2}\right) \times \mathbb{R}^{2} \backslash \mathcal{U}^{\mathrm{c}} ;
\end{aligned}
$$

i.e. $\mathcal{U}^{\mathrm{c}}$ is the set of Cauchy problems for which there is a unique solution, and $\mathcal{M}^{\mathrm{c}}$ is its complement.

TheOrem 3.2. $\mathcal{U}^{\mathrm{c}}$ is $\boldsymbol{\Pi}_{2}^{0}$-complete.

Proof. We show that $\mathcal{U}^{\mathrm{c}}$ is $\boldsymbol{\Pi}_{2}^{0}$ by showing that $\mathcal{M}^{\mathrm{c}}$ is $\boldsymbol{\Sigma}_{2}^{0}$. We have

$$
\mathcal{M}^{\mathrm{c}}=\bigcup_{\varepsilon \in \mathbb{Q}^{+}} \bigcup_{q \in \mathbb{Q}} D_{q, \varepsilon}
$$

where

$$
D_{q, \varepsilon}=\left\{(F, x, y)\left|\exists \varphi_{1}, \varphi_{2} \in \mathcal{S}_{F,(x, y)}^{\mathrm{c}}\right| \varphi_{1}(q)-\varphi_{2}(q) \mid \geq \varepsilon\right\} .
$$

Thus it suffices to show that $D_{q, \varepsilon}$ is closed. So suppose that $\left\{\left(F_{n}, x_{n}, y_{n}\right)\right\}_{n}$ is a sequence of elements of $D_{q, \varepsilon}$ which converges to some $(F, x, y)$. We need 
to show that $(F, x, y) \in D_{q, \varepsilon}$. For every $n$ there exist $\varphi_{1}^{n}, \varphi_{2}^{n} \in \mathcal{S}_{F_{n},\left(x_{n}, y_{n}\right)}^{\mathrm{c}}$ such that $\left|\varphi_{1}^{n}(q)-\varphi_{2}^{n}(q)\right| \geq \varepsilon$. By throwing away finitely many elements of the sequence we can assume that $\left\|F-F_{n}\right\|_{\infty}<1$ and $\left|x-x_{n}\right|,\left|y-y_{n}\right|<1$. Therefore the $\varphi_{i}^{n}$ 's are all $C^{1}$ functions with derivative bounded by $\|F\|_{\infty}+1$ and hence they are equicontinuous. They are also pointwise bounded because $\left|\varphi_{i}^{n}(t)-y\right| \leq|t-x|\left(\|F\|_{\infty}+1\right)$. By applying twice the Ascoli-Arzelà theorem we can assume that there exist $\varphi_{1}$ and $\varphi_{2}$ such that $\lim _{n \rightarrow \infty} \varphi_{i}^{n}=\varphi_{i}$ for $i=1,2$. Clearly, $\left|\varphi_{1}(q)-\varphi_{2}(q)\right| \geq \varepsilon$ and $\varphi_{1}, \varphi_{2} \in \mathcal{S}_{F,(x, y)}^{\mathrm{c}}$ by the closedness of $\mathcal{S}^{\text {c }}$ (Lemma 2.2).

We prove that $\mathcal{U}^{\mathrm{c}}$ is $\boldsymbol{\Pi}_{2}^{0}$-hard by showing that $N_{2}$ (see 1.1) is Wadge reducible to $\mathcal{U}^{\mathrm{c}}$ by using the technique of Lemma 2.8. It suffices to take $x_{0}=y_{0}=0, x_{1}=y_{1}=1, M=1$ and map $\beta \in \mathcal{C}$ to $F_{\beta} \in C_{\mathrm{c}}\left(\mathbb{R}^{2}\right)$ which extends $G_{\beta}$ and is zero outside the square $[0,1]^{2}$. Clearly, $\beta \mapsto\left(F_{\beta}, 0,0\right)$ is continuous and hence $N_{2} \leq_{\mathrm{w}} \mathcal{U}^{\mathrm{c}}$.

Corollary 3.3. $\mathcal{U}^{\mathrm{c}}$ is comeager in $C_{\mathrm{c}}\left(\mathbb{R}^{2}\right) \times \mathbb{R}^{2}$.

Proof. $\mathcal{U}^{\mathrm{c}}$ is a dense $\mathbf{G}_{\delta}$ as it contains all $(F, x, y)$ with $F \in \operatorname{Lip}_{2}$.

COROLlaRY 3.4. $\mathcal{U}^{\mathrm{c}}$ is not a group under the operation of addition and hence not a vector space.

Proof. $C_{\mathrm{c}}\left(\mathbb{R}^{2}\right) \times \mathbb{R}^{2}=G$ with the operation of addition is a Polish group. Since $\mathcal{U}^{\mathrm{c}} \subset G$ is $\boldsymbol{\Pi}_{2}^{0}$ (i.e. $\mathbf{G}_{\delta}$ ) but not closed it is not a subgroup.

By inspecting the proof of Theorem 3.2 we can notice that we have also proved:

Theorem 3.5. For every $(x, y) \in \mathbb{R}^{2}, \mathcal{U}_{(x, y)}^{\mathrm{c}}=\left\{F \in C_{\mathrm{c}}\left(\mathbb{R}^{2}\right) \mid \exists ! \varphi(\varphi \in\right.$ $\left.\left.\mathcal{S}_{F,(x, y)}^{\mathrm{c}}\right)\right\}$ is $\boldsymbol{\Pi}_{2}^{0}$-complete.

Hence Corollaries 3.3 and 3.4 hold also with $\mathcal{U}_{(x, y)}^{c}$ in place of $\mathcal{U}^{\mathrm{c}}$; in particular, for every $(x, y) \in \mathbb{R}^{2}, \mathcal{U}_{(x, y)}^{\mathrm{c}}$ is not a vector space.

We now move on to study equations that have a unique solution everywhere.

DEFINITION 3.6. Let

$$
\begin{aligned}
\mathcal{U}_{\forall}^{\mathrm{c}} & =\left\{F \in C_{\mathrm{c}}\left(\mathbb{R}^{2}\right) \mid \forall(x, y) \in \mathbb{R}^{2}(F, x, y) \in \mathcal{U}^{\mathrm{c}}\right\}, \\
\mathcal{M}_{\exists}^{\mathrm{c}} & =C_{\mathrm{c}}\left(\mathbb{R}^{2}\right) \backslash \mathcal{U}_{\forall}^{\mathrm{c}}=\left\{F \in C_{\mathrm{c}}\left(\mathbb{R}^{2}\right) \mid \exists(x, y) \in \mathbb{R}^{2}(F, x, y) \in \mathcal{M}^{\mathrm{c}}\right\} .
\end{aligned}
$$

TheOREM 3.7. $\mathcal{U}_{\forall}^{\mathrm{c}}$ is $\boldsymbol{\Pi}_{2}^{0}$-complete.

Proof. To see that $\mathcal{U}_{\forall}^{\mathrm{c}}$ is $\boldsymbol{\Pi}_{2}^{0}$ it suffices to show that $\mathcal{M}_{\exists}^{\mathrm{c}}$ is $\boldsymbol{\Sigma}_{2}^{0}$. This follows at once from Theorem 3.2 and Lemma 1.3 by noticing that $\mathbb{R}^{2}$ is $\mathbf{K}_{\sigma}$ and $\mathcal{M}_{\exists}^{\mathrm{c}}=\operatorname{proj}_{C_{\mathrm{c}}\left(\mathbb{R}^{2}\right)}\left(\mathcal{M}^{\mathrm{c}}\right)$. 
To see that $\mathcal{U}_{\forall}^{\mathrm{c}}$ is $\boldsymbol{\Pi}_{2}^{0}$-hard inspect the second part of the proof of Theorem 3.2: notice that $\left(F_{\beta}, 0,0\right) \in \mathcal{U}^{\mathrm{c}}$ implies $\left(F_{\beta}, x, y\right) \in \mathcal{U}^{\mathrm{c}}$ for every $(x, y) \in \mathbb{R}^{2}$. Hence $F_{\beta} \in \mathcal{U}_{\forall}^{\mathrm{c}}$ if and only if $\left(F_{\beta}, 0,0\right) \in \mathcal{U}^{\mathrm{c}}$ if and only if $\beta \in N_{2}$.

The following corollaries can be obtained from Theorem 3.7 exactly as Corollaries 3.3 and 3.4 have been obtained from Theorem 3.2.

Corollary 3.8 (Orlicz [8]). $\mathcal{U}_{\forall}^{\mathrm{c}}$ is comeager in $C_{\mathrm{c}}\left(\mathbb{R}^{2}\right)$.

COROLlary 3.9. $\mathcal{U}_{\forall}^{\mathrm{c}}$ is not a group under the operation of addition and hence not a vector space.

We have $\operatorname{Lip}_{2} \cap C_{\mathrm{c}}\left(\mathbb{R}^{2}\right) \subset \mathcal{U}_{\forall}^{\mathrm{c}}$ and we know that the two sets, as already noticed by Orlicz [8], are quite far apart from the point of view of Baire category: $\operatorname{Lip}_{2} \cap C_{\mathrm{c}}\left(\mathbb{R}^{2}\right)$ is meager while $\mathcal{U}_{\forall}^{\mathrm{c}}$ is comeager. Still, the next theorem asserts that we cannot find a $\boldsymbol{\Delta}_{2}^{0}$ set containing the former and contained in the latter. In general, if $X$ is Polish, $\boldsymbol{\Gamma}$ some class of sets and $A, B \subseteq X$ are disjoint, we say that $A$ and $B$ are $\boldsymbol{\Gamma}$-inseparable if there is no $C \in \boldsymbol{\Gamma}(X)$ with $A \subseteq C$ and $B \cap C=\emptyset$. For any $n>1$ there exist disjoint $\boldsymbol{\Sigma}_{n}^{0}$ sets which are $\boldsymbol{\Delta}_{n}^{0}$-inseparable (see [5], Theorem 22.16); the following theorem exhibits an example of this phenomenon involving natural sets.

Theorem 3.10. $\operatorname{Lip}_{2} \cap C_{\mathrm{c}}\left(\mathbb{R}^{2}\right)$ and $\mathcal{M}_{\exists}^{\mathrm{c}}$ are $\boldsymbol{\Delta}_{2}^{0}$-inseparable.

Proof. $\operatorname{Lip}_{2} \cap C_{\mathrm{c}}\left(\mathbb{R}^{2}\right)$ is dense in $C_{\mathrm{c}}\left(\mathbb{R}^{2}\right)$ and we claim that $\mathcal{M}_{\exists}^{\mathrm{c}}$ is also dense. It is easy to see that for every $(x, y) \in \mathbb{R}^{2}$ and every $z \in \mathbb{R}$ there exists $G_{(x, y), z} \in C_{\mathrm{c}}\left(\mathbb{R}^{2}\right)$ such that $G_{(x, y), z}(x, y)=z$ and $\left(G_{(x, y), z}, x, y\right) \in \mathcal{M}^{\mathrm{c}}$. Therefore for any $F \in C_{\mathrm{c}}\left(\mathbb{R}^{2}\right)$ we can change $F$ in a neighborhood of $(0,0)$ so that it coincides with $G_{(0,0), F(0,0)}$ there; the functions $\widetilde{F}$ obtained this way are such that $(\widetilde{F}, 0,0) \in \mathcal{M}^{\mathrm{c}}$ and they can be as close as we wish to $F$.

Thus if $C \in \boldsymbol{\Delta}_{2}^{0}\left(C_{\mathrm{c}}\left(\mathbb{R}^{2}\right)\right)$ contains $\operatorname{Lip}_{2} \cap C_{\mathrm{c}}\left(\mathbb{R}^{2}\right)$ and is disjoint from $\mathcal{M}_{\exists}^{\mathrm{c}}$, both $C$ and its complement are dense $\mathbf{G}_{\delta}$, violating the Baire category theorem.

3.2. Uniqueness in $C\left(\mathbb{R}^{2}\right)$. When $F \in C\left(\mathbb{R}^{2}\right)$, uniqueness of solutions of Cauchy problems means obviously uniqueness of non-extendible solutions.

DeFinition 3.11. Let

$$
\begin{aligned}
\mathcal{U} & =\left\{(F, x, y) \in C\left(\mathbb{R}^{2}\right) \times \mathbb{R}^{2} \mid \exists ! \varphi \in C_{\mathrm{par}}^{1}(\mathbb{R})\left(\varphi \in \mathcal{S}_{F,(x, y)}\right)\right\} ; \\
\mathcal{M} & =C\left(\mathbb{R}^{2}\right) \times \mathbb{R}^{2} \backslash \mathcal{U} .
\end{aligned}
$$

Theorem 3.12. $\mathcal{U}$ is $\boldsymbol{\Pi}_{2}^{0}$-complete.

Proof. We show that $\mathcal{U}$ is $\boldsymbol{\Pi}_{2}^{0}$ by showing that $\mathcal{M}$ is $\boldsymbol{\Sigma}_{2}^{0}$. For any $q \in \mathbb{Q}$, $\varepsilon \in \mathbb{Q}^{+}$and $M \in \mathbb{N}$ let $D_{q, \varepsilon}^{M}$ be the set of all $(F, x, y) \in C\left(\mathbb{R}^{2}\right) \times \mathbb{R}^{2}$ such that there exist $\varphi_{1}, \varphi_{2} \in \mathcal{S}_{F,(x, y)}$ such that 


$$
\begin{aligned}
& q \in \operatorname{dom}\left(\varphi_{1}\right) \cap \operatorname{dom}\left(\varphi_{2}\right) \&\left|\varphi_{1}(q)-\varphi_{2}(q)\right| \geq \varepsilon \\
& \&\left\|\varphi_{1} \uparrow[x, q]\right\|_{\infty},\left\|\varphi_{2} \uparrow[x, q]\right\|_{\infty} \leq M
\end{aligned}
$$

where if $q \leq x$ we take $[x, q]$ to mean the interval $[q, x]$.

Then we see that $(F, x, y) \in \mathcal{M}$ is equivalent to

$$
\exists q \in \mathbb{Q} \exists \varepsilon \in \mathbb{Q}^{+} \exists M \in \mathbb{N}(F, x, y) \in D_{q, \varepsilon}^{M}
$$

and it suffices to show that $D_{q, \varepsilon}^{M}$ is closed.

To see this suppose $\left\{\left(F_{n}, x_{n}, y_{n}\right)\right\}_{n}$ is a sequence of elements of $D_{q, \varepsilon}^{M}$ converging to some $(F, x, y)$. We need to show that $(F, x, y) \in D_{q, \varepsilon}^{M}$.

Either for infinitely many $n, q<x_{n}$ or for infinitely many $n, q>x_{n}$ and hence we may assume that the same case holds for every $n$ : for definiteness say that $q>x_{n}$. For every $n$ there exist $\varphi_{1}^{n}, \varphi_{2}^{n} \in \mathcal{S}_{F_{n},\left(x_{n}, y_{n}\right)}$ such that $\left|\varphi_{1}^{n}(q)-\varphi_{2}^{n}(q)\right| \geq \varepsilon$ and $\left\|\varphi_{i}^{n} \uparrow[x, q]\right\|_{\infty} \leq M$ for every $i=1,2$.

By applying Lemma 2.5 twice we may assume that for every $n$ we have $x \in \operatorname{dom}\left(\varphi_{1}^{n}\right) \cap \operatorname{dom}\left(\varphi_{2}^{n}\right)$ and that $\lim _{n \rightarrow \infty} \varphi_{i}^{n}(x)=y$ for $i=1,2$.

Since $\mathbf{F}\left(\mathbb{R}^{2}\right)$ is compact we can assume that $\varphi_{i}^{n} \rightarrow \Gamma_{i}$ for $i=1,2$. By Lemma 2.7, $\Gamma_{i}$ is the graph of a function $\varphi_{i}^{*}$ and, since $\lim _{n \rightarrow \infty} \varphi_{i}^{n}(x)=y$, $x \in \operatorname{dom}\left(\varphi_{i}^{*}\right)$. Let $\varphi_{i}$ be the restriction of $\varphi_{i}^{*}$ to the connected component of its domain which contains $x$. Since $\left\|\varphi_{i}^{n} \uparrow[x, q]\right\|_{\infty} \leq M$ for every $n$, we also have $[x, q] \subset \operatorname{dom}\left(\varphi_{i}\right)$ and $\| \varphi_{i}\left\lceil[x, q] \|_{\infty} \leq M .\left|\varphi_{i}^{*}(q)-\varphi_{2}^{*}(q)\right| \geq \varepsilon\right.$ is immediate and completes the proof that $(F, x, y) \in D_{q, \varepsilon}^{M}$.

The second part of the proof of Theorem 3.2 shows also that $\mathcal{U}$ is $\mathbf{\Pi}_{2^{-}}^{0}$ hard. In fact, the map used there has range contained in the set of functions with support on the square $[0,1]^{2}$; for these functions the topology of $C\left(\mathbb{R}^{2}\right)$ coincides with that of $C_{\mathrm{c}}\left(\mathbb{R}^{2}\right)$ and hence the map $\beta \mapsto F_{\beta}$ is continuous also from $\mathcal{C}$ to $C\left(\mathbb{R}^{2}\right)$.

The following corollary can be obtained from Theorem 3.12 exactly as Corollaries 3.3 and 3.4 have been obtained from Theorem 3.2.

Corollary 3.13. $\mathcal{U}$ is comeager in $C\left(\mathbb{R}^{2}\right) \times \mathbb{R}^{2} \cdot \mathcal{U}$ is not a group under the operation of addition and hence not a vector space.

Also in this case by inspecting the proof of Theorem 3.12 we can notice that we have also proved:

TheOREM 3.14. For every $(x, y) \in \mathbb{R}^{2}, \mathcal{U}_{(x, y)}=\left\{F \in C_{\mathrm{c}}\left(\mathbb{R}^{2}\right) \mid \exists ! \varphi(\varphi \in\right.$ $\left.\left.\mathcal{S}_{F,(x, y)}\right)\right\}$ is $\boldsymbol{\Pi}_{2}^{0}$-complete.

Hence Corollary 3.13 holds also with $\mathcal{U}_{(x, y)}$ in place of $\mathcal{U}$; in particular, for every $(x, y) \in \mathbb{R}^{2}, \mathcal{U}_{(x, y)}$ is not a vector space.

Moreover, if $\mathcal{U}_{\forall}=\left\{F \in C\left(\mathbb{R}^{2}\right) \mid \forall(x, y) \in \mathbb{R}^{2}(F, x, y) \in \mathcal{U}\right\}$ and $\mathcal{M}_{\exists}=$ $C\left(\mathbb{R}^{2}\right) \backslash \mathcal{U}_{\forall}$ we can obtain the following results exactly as we obtained 3.7 through 3.10 . 
TheOREM 3.15. $\mathcal{U}_{\forall}$ is $\boldsymbol{\Pi}_{2}^{0}$-complete.

COROLlary 3.16. $\mathcal{U}_{\forall}$ is comeager in $C\left(\mathbb{R}^{2}\right)$. $\mathcal{U}_{\forall}$ is not a group under the operation of addition and hence not a vector space.

Theorem 3.17. $\operatorname{Lip}_{2}$ and $\mathcal{M}_{\exists}$ are $\boldsymbol{\Delta}_{2}^{0}$-inseparable.

4. Cauchy problems with a locally unique solution. A problem connected with that of uniqueness is that of local uniqueness.

Definition 4.1. Let $\mathcal{L U}^{\mathrm{c}}$ be the set of all $(F, x, y) \in C_{\mathrm{c}}\left(\mathbb{R}^{2}\right) \times \mathbb{R}^{2}$ such that

$$
\exists \varepsilon>0 \forall \varphi, \psi \in \mathcal{S}_{F,(x, y)}^{\mathrm{c}} \forall t(|t-x|<\varepsilon \Rightarrow \varphi(t)=\psi(t)) .
$$

In other words, $(F, x, y) \in \mathcal{L U}^{\mathrm{c}}$ if and only if $(x, y)$ is not a bifurcation point for the Cauchy problem $(F, x, y)$.

Notice that our restriction to $C_{\mathrm{c}}\left(\mathbb{R}^{2}\right)$ in this case does not detract from generality: in fact, as the name suggests, when studying local uniqueness only the "local" behavior of $F$ around $(x, y)$ is relevant and thus any $F \in$ $C\left(\mathbb{R}^{2}\right)$ can be replaced by a $G \in C_{\mathrm{c}}\left(\mathbb{R}^{2}\right)$ which coincides with $F$ in a neighborhood of $(x, y)$.

TheOrem 4.2. $\mathcal{L U}^{\mathrm{c}}$ is $\boldsymbol{\Sigma}_{3}^{0}$-complete.

Proof. $(F, x, y) \in \mathcal{L U}^{c}$ is equivalent to

$$
\exists \varepsilon \in \mathbb{Q}^{+} \forall \delta \in \mathbb{Q}^{+} \forall q \in \mathbb{Q}\left(|q-x|<\varepsilon \Rightarrow(F, x, y) \notin D_{q, \delta}\right),
$$

where $D_{q, \delta}$ has been defined in the first part of the proof of Theorem 3.2. In that proof we showed that $D_{q, \delta}$ is closed and hence $\mathcal{L U}^{\mathrm{c}}$ is $\boldsymbol{\Sigma}_{3}^{0}$.

To show that $\mathcal{L U}^{\mathrm{c}}$ is $\boldsymbol{\Sigma}_{3}^{0}$-hard we will prove that $S_{3}^{\prime} \leq_{\mathrm{W}} \mathcal{L U}^{\mathrm{c}}$ where $S_{3}^{\prime}=\left\{\alpha \in 2^{\mathbb{N} \times \mathbb{N}} \mid \forall^{\infty} n \exists^{\infty} m \alpha(n, m)=0\right\}$. This is not one of the standard $\boldsymbol{\Sigma}_{3}^{0}$-complete sets but is a variant of $S_{3}$ (see 1.1) which can be easily Wadge reduced to it.

If $\alpha \in 2^{\mathbb{N} \times \mathbb{N}}$ we need to define $F_{\alpha} \in C_{\mathrm{c}}\left(\mathbb{R}^{2}\right)$. We pick $a_{n}$ 's and $b_{n}$ 's such that $0<b_{n+1}<a_{n}<b_{n}<1$ and $\lim a_{n}=\lim b_{n}=0$. Let $T_{n}=$ $\left[a_{n}, b_{n}\right] \times[0,1]$. We define $F_{\alpha}=0$ outside $\bigcup_{n} T_{n}$. For every $n$ we denote by $\beta_{n}$ the element of $\mathcal{C}$ defined by $\beta_{n}(m)=\alpha(n, m)$. By Lemma 2.8 for every $n$ there exists a continuous function $G_{n}$ defined on $T_{n}$, satisfying $\left\|G_{n}\right\|_{\infty} \leq 2^{-n}, G_{n}=0$ on the boundary of $T_{n}$ and such that the Cauchy problem $\left(G_{n}, a_{n}, 0\right)$ has a unique solution if and only if $\beta_{n} \in N_{2} . F_{\alpha}$ coincides with $G_{n}$ on $T_{n}$. The map $\alpha \mapsto F_{\alpha}$ is continuous because $\left\|G_{n}\right\|_{\infty} \rightarrow 0$. This fact also ensures the continuity of $F_{\alpha}$ at $(0, u)$ for any $u \in[0,1]$.

The fact that $\alpha \in S_{3}^{\prime}$ is equivalent to $\left(F_{\alpha}, 0,0\right) \in \mathcal{L U}^{\mathrm{c}}$ follows easily from the construction. 
COROLLARY 4.3. $\mathcal{L U}^{\mathrm{c}}$ is comeager and not a group under the operation of addition, and hence not a vector space.

Proof. The first assertion follows from $\mathcal{U}^{\mathrm{c}} \subset \mathcal{L U}^{\mathrm{c}}$ and Corollary 3.3. The second assertion follows from the first by the fact that $\mathcal{L U}^{\mathrm{c}}$ is Borel and by the property of Borel non-meager subgroups mentioned in Section 1.1.

Our proofs establish also the following theorem:

Theorem 4.4. For every $(x, y) \in \mathbb{R}^{2}, \mathcal{L U}_{(x, y)}^{\mathrm{c}}=\left\{F \in C_{\mathrm{c}}\left(\mathbb{R}^{2}\right) \mid(F, x, y)\right.$ $\left.\in \mathcal{L U}^{\mathrm{c}}\right\}$ is $\boldsymbol{\Sigma}_{3}^{0}$-complete, comeager and not a subgroup.

In this case it does not make sense to study the set of differential equations such that for every $(x, y) \in \mathbb{R}^{2}$ we have a Cauchy problem in $\mathcal{L U}^{\mathrm{c}}$ : indeed, this set obviously coincides with $\mathcal{U}_{\forall}^{\mathrm{c}}$.

\section{Cauchy problems with global solutions}

DeFINITION 5.1. Let

$$
\mathcal{G}=\left\{(F, x, y) \in C\left(\mathbb{R}^{2}\right) \times \mathbb{R}^{2} \mid \exists \varphi \in \mathcal{S}_{F,(x, y)}(\operatorname{dom}(\varphi)=\mathbb{R})\right\},
$$

i.e. $\mathcal{G}$ is the set of Cauchy problems which have a global solution.

Definition 5.2. Let $\mathcal{G}_{\forall}=\left\{F \in C\left(\mathbb{R}^{2}\right) \mid \forall(x, y) \in \mathbb{R}^{2}(F, x, y) \in \mathcal{G}\right\}$.

To simplify a bit the notation it will be convenient to study the following two sets in place of $\mathcal{G}$.

DEFINITION 5.3. Let

$$
\mathcal{G}^{+}=\left\{(F, x, y) \in C\left(\mathbb{R}^{2}\right) \times \mathbb{R}^{2} \mid \exists \varphi \in \mathcal{S}_{F,(x, y)}(\operatorname{dom}(\varphi) \supset[x,+\infty))\right\},
$$

i.e. $\mathcal{G}^{+}$is the set of Cauchy problems which have a solution with domain unbounded above.

$\mathcal{G}^{-}$is defined similarly with $(-\infty, x]$ in place of $[x,+\infty)$ and is the set of Cauchy problems which have a solution with domain unbounded below.

$\mathcal{G}^{+}$and $\mathcal{G}^{-}$are homeomorphic via the map $(F, x, y) \mapsto(G,-x, y)$, where $G(t, u)=-F(-t, u)$, and $\mathcal{G}=\mathcal{G}^{+} \cap \mathcal{G}^{-}$. Therefore to establish upper bounds for the complexity of $\mathcal{G}$ it suffices to establish upper bounds for the complexity of $\mathcal{G}^{+}$(the classes we use to classify the complexity of sets are closed under homeomorphisms and finite intersections).

5.1. Globality in presence of uniqueness. We begin the study of $\mathcal{G}$ by considering the set $\mathcal{G} \cap \mathcal{U}$, i.e. the existence of global solutions of the Cauchy problem when uniqueness holds as well. In this case the study is simpler than in the general case and the complexity of $\mathcal{G} \cap \mathcal{U}$ is lower than that of $\mathcal{G}$.

The study of $\mathcal{G} \cap \mathcal{U}$ can be carried on in two different Polish spaces: in $C\left(\mathbb{R}^{2}\right) \times \mathbb{R}^{2}$, but also in $\mathcal{U}$, since we showed in Theorem 3.12 that the latter is $\mathbf{G}_{\delta}$ in a Polish space. The results we will obtain are not affected by the 
setting we choose and, for the sake of definiteness, we study $\mathcal{G} \cap \mathcal{U}$ as a subset of $\mathcal{U}$.

Theorem 5.4. $\mathcal{G} \cap \mathcal{U}$ is $\boldsymbol{\Pi}_{2}^{0}$-complete in $\mathcal{U}$.

Proof. We begin by showing that $\mathcal{G} \cap \mathcal{U}$ is $\boldsymbol{\Pi}_{2}^{0}$. By the remarks after Definition 5.3 it suffices to show that $\mathcal{G}^{+} \cap \mathcal{U}$ is $\boldsymbol{\Pi}_{2}^{0}$. We will actually show that $\mathcal{U} \backslash \mathcal{G}^{+}$is $\boldsymbol{\Sigma}_{2}^{0}$.

Notice that $(F, x, y) \in \mathcal{U} \backslash \mathcal{G}^{+}$if and only if the unique element of $\mathcal{S}_{F,(x, y)}$ has a vertical asymptote to the right of $x$. Therefore $(F, x, y) \in \mathcal{U} \backslash \mathcal{G}^{+}$is equivalent to the existence of $q \in \mathbb{Q}$ such that for all $M \in \mathbb{N}$,

$x+1 \leq q \& \exists r \in \mathbb{R} \exists \varphi \in \mathcal{S}_{F,(x, y)}(x \leq r \leq q \& r \in \operatorname{dom}(\varphi) \&|\varphi(r)| \geq M)$.

We will show that, for any $q \in \mathbb{Q}$ and $M \in \mathbb{N}$, the set $R_{q, M}$ of $(F, x, y)$ satisfying the above condition is closed in $\mathcal{U}$.

Suppose that $\left\{\left(F_{n}, x_{n}, y_{n}\right)\right\}_{n}$ is a sequence of elements of $R_{q, M}$ which converges to $(F, x, y)$. For every $n$ let $\varphi_{n}$ and $r_{n}$ witness $\left(F_{n}, x_{n}, y_{n}\right) \in$ $R_{q, M}$. By extracting a subsequence we may assume that there exists $r=$ $\lim _{n \rightarrow \infty} r_{n}$. Let $\Gamma_{n}$ be the graph of $\varphi_{n}$. As $\mathbf{F}\left(\mathbb{R}^{2}\right)$ is compact we may assume that $\Gamma_{n} \rightarrow \Gamma \in \mathbf{F}\left(\mathbb{R}^{2}\right)$. By Lemma $2.7, \Gamma$ is either the graph of a function in $\mathcal{S}_{F,(x, y)}$ or a disjoint union of graphs of functions one of which is in $\mathcal{S}_{F,(x, y)}$. In any case let $\varphi \in \mathcal{S}_{F,(x, y)}$ be the function with graph contained in $\Gamma$. If $r \notin \operatorname{dom}(\varphi)$ then $\varphi$ has a vertical asymptote between $x$ and $r$. Hence there exists $\widetilde{r}$ that together with $\varphi$ witnesses $(F, x, y) \in R_{q, M}$. If $r \in \operatorname{dom}(\varphi)$ then $\Gamma_{n} \rightarrow \Gamma$ implies that we can apply Lemma 2.5 and deduce that for all but finitely many $n$ 's, $r \in \operatorname{dom}\left(\varphi_{n}\right)$ and $\lim _{n \rightarrow \infty} \varphi_{n}(r)=\varphi(r)$. Since $\varphi_{n} \rightarrow \varphi$ uniformly on compact sets, $|\varphi(r)| \geq M$, so that $r$ and $\varphi$ witness that $(F, x, y) \in R_{q, M}$.

We now prove that $\mathcal{G} \cap \mathcal{U}$ is $\Pi_{2}^{0}$-hard in $\mathcal{U}$. We will show that the complement of $\ell^{1}$ (see 1.1) is Wadge reducible to $\mathcal{G} \cap \mathcal{U}$. Given $\left(x_{n}\right) \in[0,1]^{\mathbb{N}}$ let, for every $n, y_{n}=x_{n}+2^{-n}>0$, so that $\left(x_{n}\right) \in \ell^{1}$ if and only if $\sum y_{n}<+\infty$. Let now $\varphi_{\left(x_{n}\right)}$ be a $C^{1}$ function with positive derivative defined on $\left(-\infty, \sum y_{n}\right)$ such that $\lim _{t \rightarrow-\infty} \varphi_{\left(x_{n}\right)}(t)=-\infty$ and for every $m \in \mathbb{N}$ we have $\varphi_{\left(x_{n}\right)}\left(\sum_{n=0}^{m} y_{n}\right)=m$. In particular, $\varphi_{\left(x_{n}\right)}$ is a bijection between its domain and $\mathbb{R}$ and hence has an inverse $\psi_{\left(x_{n}\right)}$ defined on $\mathbb{R}$. Let $F_{\left(x_{n}\right)} \in C\left(\mathbb{R}^{2}\right)$ be defined by

$$
F_{\left(x_{n}\right)}(t, u)=\varphi_{\left(x_{n}\right)}^{\prime}\left(\psi_{\left(x_{n}\right)}(u)\right) .
$$

Since $\partial F_{\left(x_{n}\right)} / \partial u=1$ we have $F_{\left(x_{n}\right)} \in \operatorname{Lip}_{2}$ and hence for every $(x, y) \in$ $\mathbb{R}^{2}$ the Cauchy problem $\left(F_{\left(x_{n}\right)}, x, y\right)$ has a unique solution: this solution is $\varphi_{\left(x_{n}\right)}(t+c)$ for some $c \in \mathbb{R}$ (depending on $\left.(x, y)\right)$. Hence $\left(F_{\left(x_{n}\right)}, 0,0\right) \in \mathcal{U}$ for every $\left(x_{n}\right)$ and

$$
\left(F_{\left(x_{n}\right)}, 0,0\right) \in \mathcal{G} \cap \mathcal{U} \Leftrightarrow \varphi_{\left(x_{n}\right)} \text { is defined on } \mathbb{R} \Leftrightarrow \sum y_{n}=+\infty \Leftrightarrow\left(x_{n}\right) \notin \ell^{1} \text {. }
$$


It is easy to see that we can arrange things so that the map $\left(x_{n}\right) \mapsto F_{\left(x_{n}\right)}$ is continuous.

Our proof shows that for every $(x, y) \in \mathbb{R}^{2},(\mathcal{G} \cap \mathcal{U})_{(x, y)}$ is $\Pi_{2}^{0}$-complete in $\mathcal{U}_{(x, y)}$. As we mentioned before, our result holds also if we consider $\mathcal{G} \cap \mathcal{U}$ as a subset of $C\left(\mathbb{R}^{2}\right) \times \mathbb{R}^{2}$ : in fact, a $\Pi_{2}^{0}$ subset of a $\Pi_{2}^{0}$ set (in this case $\mathcal{U}$ ) is $\boldsymbol{\Pi}_{2}^{0}$ and a $\boldsymbol{\Pi}_{2}^{0}$-hard subset of any set is $\boldsymbol{\Pi}_{2}^{0}$-hard.

Moreover, we can study $\mathcal{G} \cap\left(C^{1}\left(\mathbb{R}^{2}\right) \times \mathbb{R}^{2}\right)$ inside $C^{1}\left(\mathbb{R}^{2}\right) \times \mathbb{R}^{2}: C^{1}\left(\mathbb{R}^{2}\right)$ is a Polish space by identifying $F$ with $\left(F, \frac{\partial F}{\partial t}, \frac{\partial F}{\partial u}\right)$ and taking the product topology of the compact-open topologies on three copies of $C\left(\mathbb{R}^{2}\right)$. The proofs of the results above show that $\mathcal{G} \cap\left(C^{1}\left(\mathbb{R}^{2}\right) \times \mathbb{R}^{2}\right)$ is $\Pi_{2}^{0}$-complete in $C^{1}\left(\mathbb{R}^{2}\right) \times \mathbb{R}^{2}$.

Corollary 5.5. $\mathcal{G}_{\forall} \cap \mathcal{U}_{\forall}$ is $\boldsymbol{\Pi}_{2}^{0}$-complete (either in $\mathcal{U}_{\forall}$ or in $C\left(\mathbb{R}^{2}\right)$ ); $\mathcal{G} \cap \mathcal{U},(\mathcal{G} \cap \mathcal{U})_{(x, y)}, \mathcal{G}_{\forall} \cap \mathcal{U}_{\forall}, \mathcal{G}, \mathcal{G}_{(x, y)}$ and $\mathcal{G}_{\forall}$ are not groups under the operation of addition and hence not vector spaces; $\mathcal{G} \cap \mathcal{U}$ and $\mathcal{G}$ are comeager in $C\left(\mathbb{R}^{2}\right) \times \mathbb{R}^{2} ;(\mathcal{G} \cap \mathcal{U})_{(x, y)}, \mathcal{G}_{(x, y)}, \mathcal{G}_{\forall} \cap \mathcal{U}_{\forall}$ and $\mathcal{G}_{\forall}$ are comeager in $C\left(\mathbb{R}^{2}\right)$.

Proof. The fact that $\mathcal{G}_{\forall} \cap \mathcal{U}_{\forall}$ is $\boldsymbol{\Pi}_{2}^{0}$ follows from Theorem 5.4 exactly as the first part of Theorem 3.7 followed from Theorem 3.2. The fact that $\mathcal{G}_{\forall} \cap \mathcal{U}_{\forall}$ is $\Pi_{2}^{0}$-hard in both $\mathcal{U}_{\forall}$ and $C\left(\mathbb{R}^{2}\right)$ follows from the proof of Theorem 5.4: the map $\left(x_{n}\right) \mapsto F_{\left(x_{n}\right)}$ shows that the complement of $\ell^{1}$ (see 1.1) is Wadge reducible to $\mathcal{G}_{\forall}$ by a map whose range is contained in $\mathcal{U}_{\forall}$. This proves both results at once.

It is easy to see that $\mathcal{G}$ is dense within $\mathcal{U}$ and therefore Corollary 3.13 shows that $\mathcal{G} \cap \mathcal{U}$ is comeager in $C\left(\mathbb{R}^{2}\right) \times \mathbb{R}^{2}$; a similar argument shows that $\mathcal{G}_{\forall} \cap \mathcal{U}_{\forall}$ is comeager in $C\left(\mathbb{R}^{2}\right)$. The results about $\mathcal{G}$ and $\mathcal{G}_{\forall}$ follow immediately, since any set which contains a comeager set is comeager.

The assertions about the algebraic structures are immediate by the usual arguments (see Corollaries 3.4 and 4.3) and depend on the fact that all the sets are Borel in the appropriate space (for $\mathcal{G}, \mathcal{G}_{(x, y)}$ and $\mathcal{G}_{\forall}$ this will be proved in the next subsections).

5.2. Globality everywhere. Before studying $\mathcal{G}$ let us consider $\mathcal{G}_{\forall}$, which turns out to be of lower complexity.

Theorem 5.6. $\mathcal{G}_{\forall}$ is $\boldsymbol{\Pi}_{3}^{0}$-complete in $C\left(\mathbb{R}^{2}\right)$.

Proof. We begin by showing that $\mathcal{G}_{\forall}$ is $\boldsymbol{\Pi}_{3}^{0}$. First of all notice that $F \in \mathcal{G}_{\forall}$ if and only if

$$
\forall(x, y) \in \mathbb{R}^{2} \exists \varphi \in \mathcal{S}_{F,(x, y)}(\operatorname{dom}(\varphi) \supset[x-1, x+1]) .
$$

In fact, if (1) holds then $\varphi_{1} \in \mathcal{S}_{F,(x, y)}$ defined on $[x-1, x+1]$ can be connected to $\psi_{1} \in \mathcal{S}_{F,\left(x+1, \varphi_{1}(x+1)\right)}$ defined on $[x+1, x+2]$ and symmetrically on the left side to get $\varphi_{2} \in \mathcal{S}_{F,(x, y)}$ defined on $[x-2, x+2]$. Iterating this procedure we get $\varphi \in \mathcal{S}_{F,(x, y)}$ defined on $\mathbb{R}$. 
Now observe that condition (1) is in turn equivalent to

$$
\forall(q, r) \in \mathbb{Q}^{2} \exists \varphi \in \mathcal{S}_{F,(q, r)}(\operatorname{dom}(\varphi) \supset[q-1, q+1]) .
$$

To see this suppose $(2)$ holds. For any $(x, y) \in \mathbb{R}^{2}$ let $\varphi \in \mathcal{S}_{F,(x, y)}$. Let $q \in \mathbb{Q}$ be such that $\varphi(q)$ is defined and $x \leq q$; let $r_{0}, r_{1} \in \mathbb{Q}$ be such that $r_{0} \leq \varphi(q) \leq r_{1}$. By our hypothesis for $i=0,1$ there exists $\varphi_{i} \in \mathcal{S}_{F,\left(q, r_{i}\right)}$ with $\operatorname{dom}\left(\varphi_{i}\right) \supset[q-1, q+1]$. Then either for every $t \in[q, x+1]$ we have $\varphi_{0}(t) \leq \varphi(t) \leq \varphi_{1}(x)$ (and in this case $\operatorname{dom}(\varphi) \supset[x, x+1]$ ) or for some $s \in[q, x+1]$ and $i<2$ we have $\varphi(s)=\varphi_{i}(s)$. In the latter case we have $\varphi^{\prime}(s)=F(s, \varphi(s))=F\left(s, \varphi_{i}(s)\right)=\varphi_{i}^{\prime}(s)$ and we can define $\psi$ by setting

$$
\psi(t)= \begin{cases}\varphi(t) & \text { if } t \leq s \\ \varphi_{i}(t) & \text { if } t>s\end{cases}
$$

so that $\psi \in \mathcal{S}_{F,(x, y)}$ and $\operatorname{dom}(\psi) \supset[x, q+1] \supseteq[x, x+1]$. A symmetrical argument shows that we can assume $\operatorname{dom}(\varphi) \supset[x-1, x]$. Therefore (2) implies that we can assume $\operatorname{dom}(\varphi) \supset[x-1, x+1]$ and hence that $(1)$ holds.

Since $\exists \varphi \in \mathcal{S}_{F,(q, r)}(\operatorname{dom}(\varphi) \supset[q-1, q+1])$ is equivalent to $\exists M \in \mathbb{N}$ $F \in E_{M}$, where $F \in E_{M}$ if and only if

$$
\exists \varphi \in \mathcal{S}_{F,(q, r)}\left(\|\varphi \uparrow[q-1, q+1]\|_{\infty} \leq M\right)
$$

it suffices to show that $E_{M}$ is closed. This can be done by the usual argument (see e.g. the first part of the proof of Theorem 3.12).

We now show that $\mathcal{G}_{\forall}$ is $\Pi_{3}^{0}$-hard in $C\left(\mathbb{R}^{2}\right)$. With any $\alpha \in 2^{\mathbb{N} \times \mathbb{N}}$ we will associate $F_{\alpha} \in C\left(\mathbb{R}^{2}\right)$ in such a way that

$$
\exists^{\infty} n \beta_{n} \notin N_{2} \Leftrightarrow F_{\alpha} \in \mathcal{G}_{\forall},
$$

where $\beta_{n}(m)=\alpha(n, m)$. This suffices to prove our result because

$$
P_{3}^{\prime}=\left\{\alpha \in 2^{\mathbb{N} \times \mathbb{N}} \mid \exists^{\infty} n \forall^{\infty} m \alpha(n, m)=1\right\}=\left\{\alpha \in 2^{\mathbb{N} \times \mathbb{N}} \mid \exists^{\infty} n \beta_{n} \notin N_{2}\right\}
$$

is $\Pi_{3}^{0}$-complete; this is not one of the standard $\Pi_{3}^{0}$-complete sets but is a variant of $P_{3}$ (see 1.1) which can be easily Wadge reduced to it.

Consider the vertical semi-strip $B=[-1,2] \times(-\infty, 1]$. We will define $F_{\alpha}$ on $B$ so that it is equal to 0 on its boundary and set $F_{\alpha}=0$ outside $B$.

Pick $x_{i}^{n}, y_{i}^{n}$ for $i=1,2$ and $n \in \mathbb{N}$ so that

(a) $x_{0}^{0}=y_{1}^{0}=0$;

(b) $x_{0}^{n}<x_{1}^{n}<x_{0}^{n+1}<1$ and $\lim _{n \rightarrow \infty} x_{0}^{n}=1$;

(c) $y_{1}^{n}>y_{0}^{n}>y_{1}^{n+1}$ and $\lim _{n \rightarrow \infty} y_{0}^{n}=-\infty$.

Let $T_{n}=\left[x_{0}^{n}, x_{1}^{n}\right] \times\left[y_{0}^{n}, y_{1}^{n}\right]$ and $T=\bigcup_{n} T_{n}$. The behavior of $F_{\alpha}$ does not depend on $\alpha$ outside $T$.

On the interval $\left[y_{0}^{n}, y_{1}^{n}\right]$ we fix a strictly descending sequence $u_{0}^{n}=$ $y_{1}^{n}, u_{1}^{n}, u_{2}^{n}, \ldots$ which converges to $y_{0}^{n}$. Outside $T$ we draw $C^{1}$ curves in the plane according to the following rules (see Figure 1): 
- two distinct curves never intersect;

- the curve $\varphi_{0}^{n}$ starts with slope 0 from $\left(x_{1}^{n}, y_{1}^{n}\right)$, the upper right corner of $T_{n}$, and reaches some point $\left(2, \varphi_{0}^{n}(2)\right)$ on the right side of $B$, with slope 0 ;

- the curve $\varphi_{i+1}^{n}$ starts with slope 0 from $\left(x_{1}^{n}, u_{i+1}^{n}\right)$ on the right side of $T_{n}$ and reaches $\left(x_{0}^{n+1}, u_{i}^{n+1}\right)$ on the left side of $T_{n+1}$, with slope 0 ;

- the curve $\psi^{n}$ starts with slope 0 from $\left(x_{1}^{n}, y_{0}^{n}\right)$, the lower right corner of $T_{n}$, and reaches $\left(x_{0}^{n+1}, y_{0}^{n+1}\right)$, the lower left corner of $T_{n+1}$, with slope 0 .

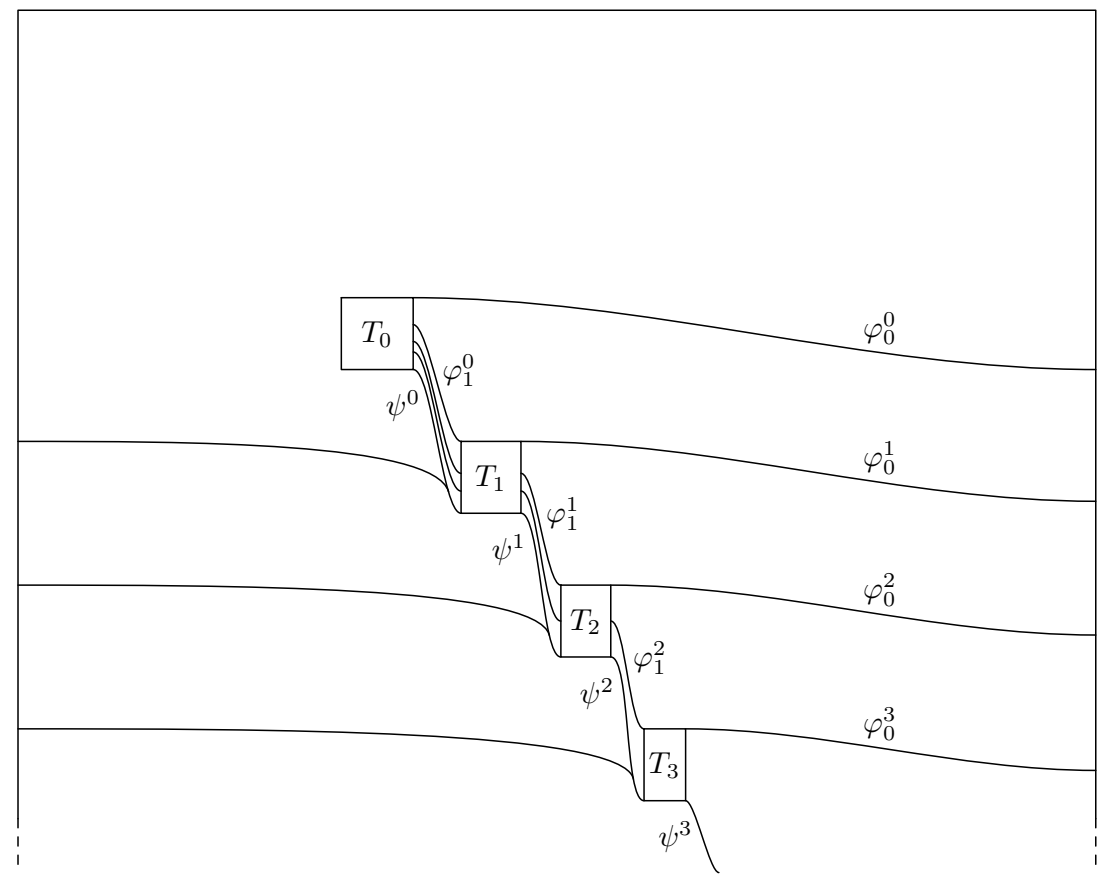

Fig. 1

$F_{\alpha}$ is defined on $\mathbb{R}^{2} \backslash T$ so that all these curves are (portions of) solutions of the differential equation given by $F_{\alpha}$. Moreover, there are no solutions of that differential equation which pass through one point on the curve $\psi^{n}$ and then move to the right of it. Similarly, there are no solutions which pass through one point on the curve $\varphi_{1}^{n}$ and then move to the right of it. Define $F_{\alpha}$ on the left of the vertical "barrier" consisting of the $\psi^{n}$ 's, so that through each point left of this barrier there exists a solution of the differential equation which reaches some point on this barrier. We furthermore want that $F_{\alpha}=0$ on the boundary of each $T_{n}$. All this can be attained by defining $F_{\alpha}$ on the union of the graphs of the curves, on appropriate neighborhoods to 
the right of the $\psi^{n}$ 's and of the $\varphi_{1}^{n}$ 's, on the portion of the plane to the left of the barrier, and on the boundaries of the $T_{n}$ 's, in the appropriate way and then using the Tietze extension theorem.

Now that we explained how to define $F_{\alpha}$ outside $T$ and the behavior of its solutions in that portion of the plane, we define $F_{\alpha}$ inside each $T_{n}$ using Lemma 2.8 (with $M=1$ and $\beta=\beta_{n}$ ). This completes the construction of $F_{\alpha}$ on $T_{n}$.

We claim that, regardless of $\alpha$, for any $n$ and $u \in\left(y_{0}^{n}, y_{1}^{n}\right]$ we have $\left(F_{\alpha}, x_{1}^{n}, u\right) \in \mathcal{G}^{+}$. To prove this it suffices to show that there exists a solution through $\left(x_{1}^{n}, u\right)$ which reaches the right side of $B$. Notice that either $u=$ $y_{1}^{n}$, so that $\varphi_{0}^{n}$ witnesses $\left(F_{\alpha}, x_{1}^{n}, u\right) \in \mathcal{G}^{+}$, or there exists $i$ such that $u \in$ $\left[u_{i+1}^{n}, u_{i}^{n}\right)$. We proceed by induction on $i$. Suppose for every $j<i, n$ and $u \in\left[u_{j+1}^{n}, u_{j}^{n}\right)$ we have $\left(F_{\alpha}, x_{1}^{n}, u\right) \in \mathcal{G}^{+}$. If $u \in\left[u_{i+1}^{n}, u_{i}^{n}\right)$ then there exists a solution through $\left(x_{1}^{n}, u\right)$ whose graph lies between $\varphi_{i+1}^{n}$ and $\varphi_{i}^{n}$. If this solution does not reach the boundary of $B$ (and hence witnesses $\left(F_{\alpha}, x_{1}^{n}, u\right) \in$ $\left.\mathcal{G}^{+}\right)$it reaches the left side of $T_{n+1}$ at some point $\left(x_{0}^{n+1}, v\right)$ with $v \geq u_{i}^{n+1}$. So it suffices to show that $\left(F_{\alpha}, x_{0}^{n+1}, v\right) \in \mathcal{G}^{+}$. Since $F_{\alpha}$ is non-negative inside $T_{n+1}$ there exists a solution through $\left(x_{0}^{n+1}, v\right)$ which exits $T_{n+1}$ on the right side at $\left(x_{1}^{n+1}, w\right)$ with $w \geq v \geq u_{i}^{n+1}$. Now it suffices to show that $\left(F_{\alpha}, x_{1}^{n+1}, w\right) \in \mathcal{G}^{+}$. Since $w \in\left[u_{j+1}^{n+1}, u_{j}^{n+1}\right)$ for some $j<i$ this follows from the induction hypothesis.

We are now ready to show that $F_{\alpha} \in \mathcal{G}_{\forall}$ if and only if $\alpha \in P_{3}^{\prime}$.

If $\exists^{\infty} n, \beta_{n} \notin N_{2}$, for infinitely many $n$ 's multiplicity occurs at the lower left corner $\left(x_{0}^{n}, y_{0}^{n}\right)$ of $T_{n}$. If $(x, y)$ lies to the left of the barrier there exists a solution of the differential equation that leads to the barrier before some $T_{n_{0}}$. By hypothesis, for some $n>n_{0}$ multiplicity occurs and hence after following $\psi^{n_{0}}, \psi^{n_{0}+1}, \ldots, \psi^{n-1}$ to reach the lower left corner $\left(x_{0}^{n}, y_{0}^{n}\right)$ of $T_{n}$ we have at least one solution which exits $T_{n}$ at $\left(x_{1}^{n}, u\right)$ with $u \in\left(y_{0}^{n}, y_{1}^{n}\right]$. By the claim we proved above, $\left(F_{\alpha}, x_{1}^{n}, u\right) \in \mathcal{G}^{+}$and hence $\left(F_{\alpha}, x, y\right) \in \mathcal{G}$. The argument to show that we have global solutions also for points lying to the right of our barrier is simpler. Therefore $F_{\alpha} \in \mathcal{G}_{\forall}$.

Now suppose that for all but finitely many $n$ 's we have $\beta_{n} \in N_{2}$. Let $n_{0}$ be such that for every $n \geq n_{0}$ we have $\beta_{n} \in N_{2}$ and consider the Cauchy problem given by $\left(F_{\alpha}, x_{0}^{n_{0}}, y_{0}^{n_{0}}\right)$. Since in each $T_{n}$ to the right of the initial condition we have uniqueness, the only solution goes through the various $\psi^{n}$ and hence has a vertical asymptote at $t=1$. Therefore $\left(F_{\alpha}, x_{0}^{n_{0}}, y_{0}^{n_{0}}\right) \notin \mathcal{G}$ and hence $F_{\alpha} \notin \mathcal{G}_{\forall}$.

5.3. Globality of solutions to Cauchy problems. We now turn to $\mathcal{G}$. The existence of arbitrarily long solutions of a Cauchy problem does not, in general, suffice to ensure the existence of a global solution of that problem. For example, we can construct an $F$ such that $\mathcal{S}_{F,(-1,0)} \supset\left\{\varphi_{n} \mid n \in \mathbb{N}\right\}$ 
with $\operatorname{dom}\left(\varphi_{n}\right)=(-\infty, n), \lim _{t \rightarrow 0^{-}} \varphi_{0}(t)=-\infty$, and $\lim _{t \rightarrow n^{-}} \varphi_{n}(t)=+\infty$ when $n \geq 1$, so that the Cauchy problem given by $(F,-1,0)$ has no global solutions (see Figure 2).

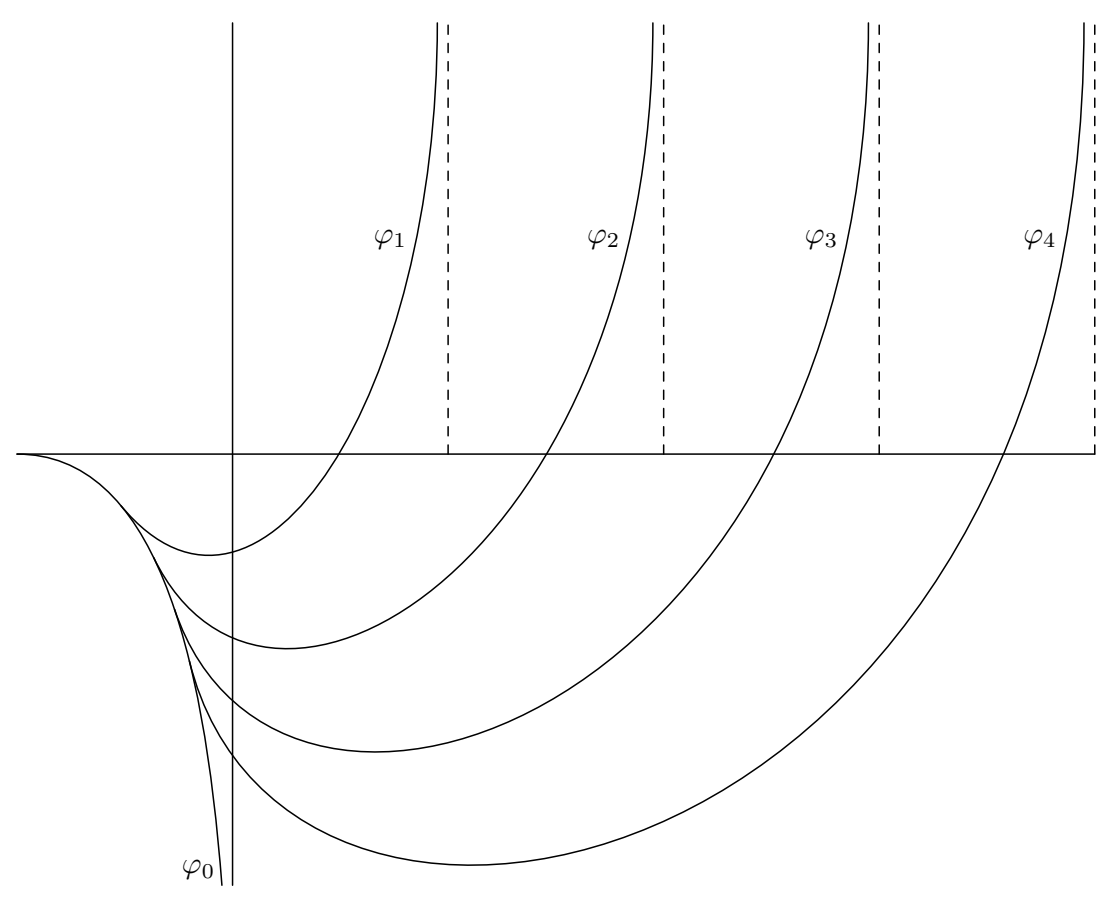

Fig. 2

LEMMA 5.7. $\mathcal{G}$ is $\boldsymbol{\Sigma}_{4}^{0}$-hard.

Proof. We will show that $S_{4}$ (see 1.1) is Wadge reducible to $\mathcal{G}$. Given $\alpha \in 2^{\mathbb{N} \times \mathbb{N}}$ we will define $F_{\alpha}$ so that $\alpha \in S_{4}$ if and only if $\left(F_{\alpha},-1,0\right) \in \mathcal{G}$.

As in the proof of Theorem 5.6, $F_{\alpha}$ will be independent of $\alpha$ outside a countable union of rectangles $T=\bigcup_{n} T_{n}$, but now $T_{n}=[n+1 / 4, n+3 / 4]$ $\times[0,1 / 2]$. Outside $T$ we draw $C^{1}$ curves in the plane according to the following rules (see Figure 3):

- the curve $\chi$ starts from $(-1,0)$ and has $t=0$ as vertical asymptote to $+\infty$;

- the curve $\chi^{n}$ starts from the point $\left(-2^{-n}, \chi\left(-2^{-n}\right)\right)$ on $\chi$, and reaches $(n+1 / 4,0)$, the lower left corner of $T_{n}$, with slope 0 ;

- the curve $\psi^{n}$ starts with slope 0 from $(n+3 / 4,0)$, the lower right corner of $T_{n}$, and has $t=n+1$ as vertical asymptote to $-\infty$;

- the curve $\varphi_{i}^{n}$ starts with slope 0 from $\left(n+3 / 4,2^{-i+1}\right)$ on the right side of $T_{n}$ and reaches $(n+1+1 / 4,0)$, the lower left corner of $T_{n+1}$, with slope 0 ; at this point it merges with $\chi^{n+1}$ and the other $\varphi_{j}^{n}$ 's. 


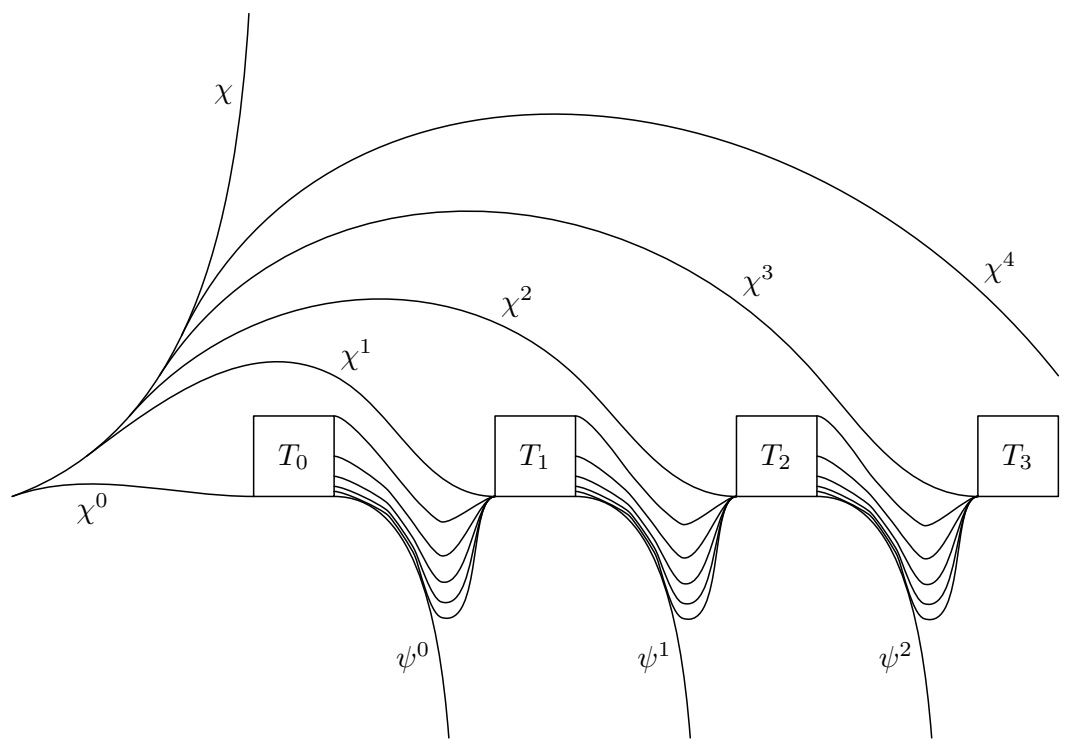

Fig. 3

Obviously, all these curves should be arranged so that at their intersections they have the same slope and so that the function describing their derivatives is continuous; this, in particular, requires that $\varphi_{i}^{n} \rightarrow \psi^{n}$ pointwise on the interval $[n+3 / 4, n+1)$.

Now we define $F_{\alpha}$ on $\mathbb{R}^{2} \backslash T$ by requiring that all these curves are (portions of) solutions of the differential equation given by $F_{\alpha}$. We make sure we avoid the existence of solutions of the differential equation which pass through one point on the curve $\psi^{n}$ and then move to the right of it. Similarly, there should be no bifurcation points on the $\chi^{n}$ 's or the $\varphi_{i}^{n}$ 's. There should also be a solution moving from $(-1,0)$ to the left to $-\infty$. This can be attained as in the proof of Theorem 5.6.

The definition of $F_{\alpha}$ within each $T_{n}$ follows again the same ideas employed in the proof of Theorem 5.6 and we are left with showing that this construction actually works.

First suppose that $\alpha$ is such that $\forall^{\infty} n \forall^{\infty} m \alpha(n, m)=0$. This means that for all but finitely many $n$ 's we have multiplicity within $T_{n}$. Let $n_{0}$ be such that this happens for every $n \geq n_{0}$. From $(-1,0)$ follow $\chi$ until $\chi^{n_{0}}$ splits and follow the latter to $\left(n_{0}+1 / 4,0\right)$. Now avoid the possibility of being led to an asymptote by $\psi^{n_{0}}$ by exiting from $T_{n_{0}}$ above some $\varphi_{i}^{n_{0}}$. This can be repeated for all the following $T_{n}$ 's thereby constructing a global solution of the Cauchy problem.

If $\alpha$ is such that for infinitely many $n$ 's we have local uniqueness within $T_{n}$, then no matter which $\chi^{n_{0}}$ we will pick to avoid being led to a vertical 
asymptote by $\chi$, we will be led to some other vertical asymptote by $\psi^{n}$ where $n \geq n_{0}$ is the first such that uniqueness holds in $T_{n}$.

Notice that Lemma 5.7 and Theorem 5.6 show that the complexities of $\mathcal{G}$ and $\mathcal{G}_{\forall}$ are different. Contrast this with the fact that the complexities of $\mathcal{U}$ and $\mathcal{U}_{\forall}$ are the same (Theorems 3.12 and 3.15).

Our proof shows also that for every $(x, y) \in \mathbb{R}^{2}, \mathcal{G}_{(x, y)}$ is $\boldsymbol{\Sigma}_{4}^{0}$-hard.

The obstacle to the existence of a global solution of the Cauchy problem whose solutions are depicted in Figure 2 is that $\lim _{n \rightarrow \infty} \varphi_{n}(0)=-\infty$, i.e. the $\varphi_{n}$ 's drop arbitrarily low. The next lemma shows that if this does not happen then a global solution does exist. Let us first introduce some helpful notation and prove an easy proposition.

Definition 5.8. If $F \in C\left(\mathbb{R}^{2}\right)$ and $(x, y) \in \mathbb{R}^{2}$ let

$$
\mathcal{S}_{F,(x, y)}^{+}=\left\{\varphi\left\lceil[x,+\infty) \mid \varphi \in \mathcal{S}_{F,(x, y)}\right\} .\right.
$$

In other words, $\mathcal{S}_{F,(x, y)}^{+}$is the set of $\varphi \in C^{1}([x, b))$ for some $b$ such that $x<b \leq+\infty$ satisfying $\varphi(x)=y, \varphi^{\prime}(t)=F(t, \varphi(t)$ ) for every $t \in[x, b$ ) (in $x$ we are considering the right derivative), and which are non-extendible to the right.

DEFinition 5.9. If $\left\{\varphi_{n}\right\}_{n}$ is a sequence of real-valued functions defined on subsets of $\mathbb{R}$ let $\inf _{n} \varphi_{n}$ be the function defined on a subset of $\bigcup_{n} \operatorname{dom}\left(\varphi_{n}\right)$ by $\inf _{n} \varphi_{n}(t)=\inf \left\{\varphi_{n}(t) \mid t \in \operatorname{dom}\left(\varphi_{n}\right)\right\}$ (the function is defined only if the inf is greater then $-\infty)$.

DeFinition 5.10. If $\varphi$ is a real-valued function defined on an interval in $\mathbb{R}$ and $b=\sup (\operatorname{dom}(\varphi))$ we denote $\lim _{t \rightarrow b-} \varphi(t)$ by $\lim _{+} \varphi$.

DeFinition 5.11. If $\varphi$ and $\psi$ are two real-valued functions defined on subsets of $\mathbb{R}$ we write $\varphi \leq \psi$ if $\varphi(t) \leq \psi(t)$ whenever $t \in \operatorname{dom}(\varphi) \cap \operatorname{dom}(\psi)$. We also define two functions $\varphi \vee \psi$ and $\varphi \wedge \psi$ with domain $\operatorname{dom}(\varphi) \cup \operatorname{dom}(\psi)$. Both functions coincide with $\varphi$ on $\operatorname{dom}(\varphi) \backslash \operatorname{dom}(\psi)$ and with $\psi$ on $\operatorname{dom}(\psi) \backslash$ $\operatorname{dom}(\varphi)$. If $t \in \operatorname{dom}(\varphi) \cap \operatorname{dom}(\psi)$ then $(\varphi \vee \psi)(t)=\max (\varphi(t), \psi(t))$ and $(\varphi \wedge \psi)(t)=\min (\varphi(t), \psi(t))$.

Proposition 5.12. If $F \in C\left(\mathbb{R}^{2}\right),(x, y) \in \mathbb{R}^{2}$ and $\varphi, \psi \in \mathcal{S}_{F,(x, y)}^{+}$are such that $\operatorname{dom}(\varphi) \subseteq \operatorname{dom}(\psi)$ then:

(1) $\lim _{+} \varphi \neq-\infty$ implies $\varphi \wedge \psi \in \mathcal{S}_{F,(x, y)}^{+}$;

(2) $\lim _{+} \varphi \neq+\infty$ implies $\varphi \vee \psi \in \mathcal{S}_{F,(x, y)}^{+}$.

Proof. The condition on $\lim _{+} \varphi$ ensures that $\varphi \wedge \psi($ resp. $\varphi \vee \psi$ ) is continuous. The fact that whenever $\varphi(t)=\psi(t)$ we also have $\varphi^{\prime}(t)=\psi^{\prime}(t)$ immediately yields the conclusion. 
The next lemma gives a way to build new solutions of an ODE out of old ones.

Lemma 5.13. Let $(F, x, y) \in C\left(\mathbb{R}^{2}\right) \times \mathbb{R}^{2}$ and let $\left\{\varphi_{n}\right\}_{n}$ be a sequence of elements of $\mathcal{S}_{F,(x, y)}^{+}$such that $\lim _{+} \varphi_{n} \neq-\infty$ for every $n$. Let $T \in(x,+\infty]$ be such that:

(1) $[x, T) \subseteq \bigcup_{n} \operatorname{dom}\left(\varphi_{n}\right)$;

(2) for every $t \in[x, T)$ we have $\inf _{n} \varphi_{n}(t)>-\infty$;

(3) if $T<+\infty$, then $\inf _{n} \varphi_{n}(T)=-\infty$.

Then $\inf _{n} \varphi_{n}\left\lceil[x, T) \in \mathcal{S}_{F,(x, y)}^{+}\right.$. In particular, if $T=+\infty$ then $\inf _{n} \varphi_{n} \in$ $\mathcal{S}_{F,(x, y)}^{+}$.

Proof. Replacing $\varphi_{n}$ by $\varphi_{0} \wedge \ldots \wedge \varphi_{n}$ (and using Proposition 5.12) we may assume that $\operatorname{dom}\left(\varphi_{n}\right) \subseteq \operatorname{dom}\left(\varphi_{n+1}\right)$ and $\varphi_{n} \geq \varphi_{n+1}$. Therefore the graph of $\inf _{n} \varphi_{n}$ is the limit within $\mathbf{F}\left(\mathbb{R}^{2}\right)$ of the graphs of the $\varphi_{n}$ 's. The conclusion follows by Lemma 2.7.

We now define two subsets of $\mathcal{G}^{+}$.

Definition 5.14. For $(F, x, y) \in C\left(\mathbb{R}^{2}\right) \times \mathbb{R}^{2}$ let $(F, x, y) \in \mathcal{H}_{-}$if and only if there are $N, h \in \mathbb{N}$ such that

$$
\forall n \exists \varphi_{n} \in \mathcal{S}_{F,(x, y)}^{+}\left([x, x+N+n] \subseteq \operatorname{dom}\left(\varphi_{n}\right) \& \| \varphi_{n}\left\lceil[x, x+N] \|_{\infty} \leq h\right)\right.
$$

and

$\neg \exists \varphi \in \mathcal{S}_{F,(x, y)}^{+}\left([x, x+N] \subseteq \operatorname{dom}(\varphi) \&\|\varphi \uparrow[x, x+N]\|_{\infty} \leq h \& \lim _{+} \varphi=-\infty\right)$.

In other words, $(F, x, y) \in \mathcal{H}_{-}$if and only if there exists a rectangle of base $[x, x+N]$ and height $[-h, h]$ such that $(F, x, y)$ admits partial solutions of arbitrary length which are trapped inside the rectangle, but no solution trapped inside the rectangle has a vertical asymptote going to $-\infty$.

$\mathcal{H}_{+}$is defined analogously by asking that no solution trapped inside the rectangle has a vertical asymptote going to $+\infty$.

LEMmA 5.15. $\mathcal{H}_{-} \cup \mathcal{H}_{+} \subseteq \mathcal{G}^{+}$.

Proof. By the symmetry of the definitions of $\mathcal{H}_{-}$and $\mathcal{H}_{+}$it suffices to prove $\mathcal{H}_{-} \subseteq \mathcal{G}^{+}$. Let $(F, x, y) \in \mathcal{H}_{-}$and $N, h$ and $\left\{\varphi_{n}\right\}_{n}$ be as in the definition.

We claim that $\inf \left\{\varphi_{n}(t) \mid t \in \operatorname{dom}\left(\varphi_{n}\right)\right\}>-\infty$ for every $t \in[x,+\infty)$. If $t \in[x, x+N]$ this follows from the fact that $\varphi_{n}(t) \geq-h$ for every $n$. Suppose now, towards a contradiction, that $t>x+N$ is such that $\inf \left\{\varphi_{n}(t) \mid t \in \operatorname{dom}\left(\varphi_{n}\right)\right\}=-\infty$. Lemma 5.13 implies that for some $T \leq t$, $\varphi=\inf _{n} \varphi_{n} \uparrow[x, T)$ is an element of $\mathcal{S}_{F,(x, y)}^{+}$satisfying $[x, x+N] \subset \operatorname{dom}(\varphi)$, $\|\varphi \uparrow[x, x+N]\|_{\infty} \leq h$, and $\lim _{+} \varphi=-\infty$. This contradicts $(F, x, y) \in \mathcal{H}_{-}$ and proves the claim. 
The claim and Lemma 5.13 imply that $(F, x, y) \in \mathcal{G}^{+}$.

We now define another subset of $\mathcal{G}^{+}$.

Definition 5.16. For $(F, x, y) \in C\left(\mathbb{R}^{2}\right) \times \mathbb{R}^{2}$ let $(F, x, y) \in \mathcal{H}_{\infty}$ if and only if for all $n$ there exist $\varphi_{n}, \psi_{n} \in \mathcal{S}_{F,(x, y)}^{+}$such that

$[x, x+n] \subseteq \operatorname{dom}\left(\varphi_{n}\right) \cap \operatorname{dom}\left(\psi_{n}\right) \& \lim _{+} \varphi_{n}=+\infty \& \lim _{+} \psi_{n}=-\infty$.

In other words, $(F, x, y) \in \mathcal{H}_{\infty}$ if and only if the Cauchy problem admits both partial solutions of arbitrary length such that $\lim _{+} \varphi=+\infty$ and partial solutions of arbitrary length such that $\lim _{+} \psi=-\infty$.

LEMma 5.17. $\mathcal{H}_{\infty} \subseteq \mathcal{G}^{+}$.

Proof. Suppose $(F, x, y) \in \mathcal{H}_{\infty}$ and let $\left\{\varphi_{n}\right\}_{n}$ and $\left\{\psi_{n}\right\}_{n}$ witness this. If the domain of some $\varphi_{n}$ or $\psi_{n}$ is unbounded above we are done. Otherwise let $b_{n}$ and $d_{n}$ be such that $\operatorname{dom}\left(\varphi_{n}\right)=\left[x, b_{n}\right)$ and $\operatorname{dom}\left(\psi_{n}\right)=\left[x, d_{n}\right)$. We can suppose that $b_{n} \leq d_{n}<b_{n+1}$ for every $n$. We define inductively two sequences $\left\{\widetilde{\varphi}_{n}\right\}_{n}$ and $\left\{\widetilde{\psi}_{n}\right\}_{n}$ of elements of $\mathcal{S}_{F,(x, y)}^{+}$as follows:

$$
\begin{aligned}
\widetilde{\varphi}_{0} & =\varphi_{0} ; & \widetilde{\psi}_{0} & =\varphi_{0} \wedge \psi_{0} ; \\
\widetilde{\varphi}_{n+1} & =\widetilde{\psi}_{n} \vee\left(\widetilde{\varphi}_{n} \wedge \varphi_{n+1}\right) ; & \widetilde{\psi}_{n+1} & =\widetilde{\varphi}_{n+1} \wedge\left(\widetilde{\psi}_{n} \vee \psi_{n+1}\right) .
\end{aligned}
$$

Using Proposition 5.12 a straightforward induction shows that

(a) $\operatorname{dom}\left(\widetilde{\varphi}_{n}\right)=\operatorname{dom}\left(\varphi_{n}\right)$ and $\operatorname{dom}\left(\widetilde{\psi}_{n}\right)=\operatorname{dom}\left(\psi_{n}\right)$;

(b) $\widetilde{\varphi}_{n} \geq \widetilde{\varphi}_{n+1} \geq \widetilde{\psi}_{n+1} \geq \widetilde{\psi}_{n}$;

(c) $\lim _{+} \widetilde{\varphi}_{n}=+\infty$ and $\lim _{+} \widetilde{\psi}_{n}=-\infty$;

(d) $\widetilde{\varphi}_{n}, \widetilde{\psi}_{n} \in \mathcal{S}_{F,(x, y)}^{+}$.

If $t>x$ let $N$ be such that $t<x+N$; for every $n$ such that $t \in$ $\operatorname{dom}\left(\widetilde{\varphi}_{n}\right)$ we have $\widetilde{\varphi}_{n}(t) \geq \widetilde{\psi}_{N}(t)$. Hence $\inf \left\{\widetilde{\varphi}_{n}(t) \mid t \in \operatorname{dom}\left(\widetilde{\varphi}_{n}\right)\right\}>$ $-\infty$ and we can apply Lemma 5.13 to obtain $\widetilde{\varphi}=\inf _{n} \widetilde{\varphi}_{n} \in \mathcal{S}_{F,(x, y)}^{+}$. As $\operatorname{dom}(\widetilde{\varphi})=\bigcup_{n} \operatorname{dom}\left(\widetilde{\varphi}_{n}\right)=\bigcup_{n} \operatorname{dom}\left(\varphi_{n}\right) \supseteq \bigcup_{n}[x, x+n]$ it follows that $\operatorname{dom}(\widetilde{\varphi})=[x,+\infty)$. Therefore $(F, x, y) \in \mathcal{G}^{+}$.

$\mathcal{H}_{-} \cup \mathcal{H}_{+}$and $\mathcal{H}_{\infty}$ show two different ways in which a Cauchy problem can have a solution with domain unbounded above. These are actually the only possible ways this can happen.

Theorem 5.18. $\mathcal{G}^{+}=\mathcal{H}_{-} \cup \mathcal{H}_{+} \cup \mathcal{H}_{\infty}$.

Proof. Lemmas 5.15 and 5.17 show that $\mathcal{H}_{-} \cup \mathcal{H}_{+} \cup \mathcal{H}_{\infty} \subseteq \mathcal{G}^{+}$, so we need to prove only the reverse inclusion.

Let $(F, x, y) \in \mathcal{G}^{+}$and define

$$
\begin{aligned}
& B^{+}=\left\{b \mid \exists \varphi \in \mathcal{S}_{F,(x, y)}^{+}\left(\operatorname{dom}(\varphi)=[x, b) \& \lim _{+} \varphi=+\infty\right)\right\} \cup\{x\}, \\
& B^{-}=\left\{b \mid \exists \varphi \in \mathcal{S}_{F,(x, y)}^{+}\left(\operatorname{dom}(\varphi)=[x, b) \& \lim _{+} \varphi=-\infty\right)\right\} \cup\{x\}
\end{aligned}
$$


and set

$$
b^{+}=\sup B^{+} \quad \text { and } \quad b^{-}=\sup B^{-} .
$$

If $b^{+}=b^{-}=+\infty$ then clearly $(F, x, y) \in \mathcal{H}_{\infty}$. Now we will show that if $b^{-}<+\infty$ then $(F, x, y) \in \mathcal{H}_{-}$; a symmetric argument shows that if $b^{+}<+\infty$ then $(F, x, y) \in \mathcal{H}_{+}$and completes the proof of the theorem.

Suppose $b^{-}<+\infty$ and let $N \in \mathbb{N}$ be such that $b^{-}<x+N$. Since $(F, x, y) \in \mathcal{G}^{+}$there exists $\psi \in \mathcal{S}_{F,(x, y)}^{+}$such that $\operatorname{dom}(\psi)=[x,+\infty)$. Fix such a $\psi$ and let $h \in \mathbb{N}$ be such that $\|\psi \uparrow[x, x+N]\|_{\infty} \leq h$. We claim that $N$ and $h$ witness $(F, x, y) \in \mathcal{H}_{-}$. For every $n$ we can set $\varphi_{n}=\psi$ so that the first part of the definition (the existence of solutions of arbitrary length) is satisfied. On the other hand, the choice of $N$ guarantees that also the second part of the definition (the non-existence of solutions with $\lim _{+} \varphi=-\infty$ which are trapped inside the rectangle $\left.[x, x+N] \times[-h, h]\right)$ is satisfied.

Theorem 5.18 shows that to find an upper bound for the complexity of $\mathcal{G}^{+}$(and hence of $\mathcal{G}$ ) it suffices to find upper bounds for the complexities of $\mathcal{H}_{-}$and $\mathcal{H}_{\infty}$ (obviously, the complexity of $\mathcal{H}_{+}$is the same as that of $\mathcal{H}_{-}$). This is precisely what we are going to do, using the following technical lemma.

Lemma 5.19. (a) Fix $N, h \in \mathbb{N}$. Let $A_{N, h}^{+}$be the set of all Cauchy problems which have a solution bounded by $h$ on the interval $[x, x+N]$ with a vertical asymptote to $+\infty$ at some $\xi>x+N$, i.e.

$$
\begin{aligned}
& A_{N, h}^{+}=\left\{(F, x, y) \in C\left(\mathbb{R}^{2}\right) \times \mathbb{R}^{2} \mid \exists \varphi \in \mathcal{S}_{F,(x, y)}^{+}[\exists \xi>x+N(\operatorname{dom}(\varphi)=[x, \xi))\right. \\
& \left.\& \lim _{+} \varphi=+\infty \& \| \varphi\left\lceil[x, x+N] \|_{\infty} \leq h\right]\right\} .
\end{aligned}
$$

Similarly we define $A_{N, h}^{-}$by replacing " $\lim _{+} \varphi=+\infty$ " with " $\lim _{+} \varphi=-\infty$ ". Then both $A_{N, h}^{+}$and $A_{N, h}^{-}$are $\boldsymbol{\Sigma}_{2}^{0}$.

(b) Fix $N \in \mathbb{N}$. Let $A_{N}^{+}$be the set of all Cauchy problems which have a solution with a vertical asymptote to $+\infty$ at some $\xi>x+N$, i.e.

$$
\begin{aligned}
A_{N}^{+}=\{(F, x, y) & \in C\left(\mathbb{R}^{2}\right) \times \mathbb{R}^{2} \mid \\
\exists \varphi & \left.\in \mathcal{S}_{F,(x, y)}^{+}\left[\exists \xi>x+N(\operatorname{dom}(\varphi)=[x, \xi)) \& \lim _{+} \varphi=+\infty\right]\right\} .
\end{aligned}
$$

Similarly we define $A_{N}^{-}$by replacing " $\lim _{+} \varphi=+\infty$ " with " $\lim _{+} \varphi=-\infty$ ". Then both $A_{N}^{+}$and $A_{N}^{-}$are $\boldsymbol{\Sigma}_{2}^{0}$.

(c) Fix $M, N, h \in \mathbb{N}$, with $M \geq N$. Let $B_{N, h}^{M}$ be the set of all Cauchy problems which have a solution of length at least $M$ bounded by $h$ on the interval $[x, x+N]$, i.e. 


$$
\begin{aligned}
B_{N, h}^{M}=\{(F, x, y) & \in C\left(\mathbb{R}^{2}\right) \times \mathbb{R}^{2} \mid \\
\exists \varphi & \in \mathcal{S}_{F,(x, y)}^{+}\left[\operatorname{dom}(\varphi) \supseteq[x, x+M] \& \| \varphi\left\lceil[x, x+N] \|_{\infty} \leq h\right]\right\} .
\end{aligned}
$$

Then $B_{N, h}^{M}$ is $\boldsymbol{\Sigma}_{2}^{0}$.

Pr o of. (a) We claim that

$$
A_{N, h}^{+}=\bigcup_{b, l \in \mathbb{Q}} \bigcap_{M \in \mathbb{N}} D_{b, l}^{M}
$$

where

$$
\begin{aligned}
D_{b, l}^{M}=\left\{(F, x, y) \in C\left(\mathbb{R}^{2}\right)\right. & \times \mathbb{R}^{2} \mid \\
& \exists r \\
& \in \mathbb{R} \exists \varphi \in \mathcal{S}_{F,(x, y)}^{+}[x+N \leq r \leq x+N+l \\
& \& \| \varphi\left\lceil[x, x+N] \|_{\infty} \leq h \& r \in \operatorname{dom}(\varphi)\right. \\
& \&(\forall t \in[x, r] \varphi(t) \geq b) \& \varphi(r) \geq M]\} .
\end{aligned}
$$

In fact, if $\varphi$ witnesses $(F, x, y) \in A_{N, h}^{+}$let $\operatorname{dom}(\varphi)=[x, \xi)$ with $\xi>x+N$ so that

$$
\lim _{t \rightarrow \xi} \varphi(t)=+\infty .
$$

Then we can pick $b, l \in \mathbb{Q}$ such that $x+N+l \geq \xi$ and $b \leq \inf \{\varphi(t) \mid x \leq t$ $\leq \xi\}$. Therefore for every $M \in \mathbb{N}$ there exists $r \in[x, \xi)$ such that $\varphi(r) \geq M$. Thus $(F, x, y) \in D_{b, l}^{M}$.

Conversely suppose $(F, x, y) \in \bigcap_{M \in \mathbb{N}} D_{b, l}^{M}$ for some $b, l \in \mathbb{Q}$. Then for every $M \in \mathbb{N}$ there exist $r_{M}$ and $\varphi_{M}$ satisfying the conditions in the definition of $D_{b, l}^{M}$. As $[x+N, x+N+l]$ and $\mathbf{F}\left(\mathbb{R}^{2}\right)$ are compact, by extracting a subsequence we may assume that $\lim _{M \rightarrow+\infty} r_{M}=r \in[x+N, x+N+l]$ and $\lim _{M \rightarrow+\infty} \varphi_{M}=\Gamma \in \mathbf{F}\left(\mathbb{R}^{2}\right)$. By Lemma 2.7 there exists $\varphi \in \mathcal{S}_{F,(x, y)}^{+}$ whose graph is contained in $\Gamma$ and such that $[x, x+N] \subset \operatorname{dom}(\varphi) \subseteq[x, r)$, $\|\varphi \uparrow[x, x+N]\|_{\infty} \leq h$ and $\varphi$ is bounded below by $b$. Therefore $\varphi$ has a vertical asymptote to $+\infty$ at some $\xi \leq r$, and hence $(F, x, y) \in A_{N, h}^{+}$.

Notice that in proving $(*)$ we actually verified that $D_{b, l}^{M}$ is closed and therefore $A_{N, h}^{+}$is $\boldsymbol{\Sigma}_{2}^{0}$. The result about $A_{N, h}^{-}$is completely analogous.

(b) follows from (a) and the equalities $A_{N}^{+}=\bigcup_{h \in \mathbb{N}} A_{N, h}^{+}$and $A_{N}^{-}=$ $\bigcup_{h \in \mathbb{N}} A_{N, h}^{-}$.

(c) The argument is similar to the one used to prove (a), only simpler. Fix $M, N$ and $h$. Then

$$
B_{N, h}^{M}=\bigcup_{\ell \in \mathbb{Q}^{+}} E_{l}
$$

with 


$$
\begin{array}{r}
E_{l}=\left\{(F, x, y) \in C\left(\mathbb{R}^{2}\right) \times \mathbb{R}^{2} \mid \exists \varphi \in \mathcal{S}_{F,(x, y)}^{+}[\operatorname{dom}(\varphi) \supset[x, x+M]\right. \\
\& \| \varphi\left\lceil[x, x+M]\left\|_{\infty} \leq l \&\right\| \varphi\left\lceil[x, x+N] \|_{\infty} \leq h\right]\right\} .
\end{array}
$$

By Ascoli-Arzelà or, equivalently, by the compactness of $\mathbf{F}\left(\mathbb{R}^{2}\right)$, each $E_{l}$ is closed and hence $B_{N, h}^{M}$ is $\boldsymbol{\Sigma}_{2}^{0}$.

Lemma 5.20. $\mathcal{H}_{-}$and $\mathcal{H}_{+}$are $\boldsymbol{\Sigma}_{4}^{0}$ and $\mathcal{H}_{\infty}$ is $\boldsymbol{\Pi}_{3}^{0}$.

Pr o of. Using the notations of Lemma 5.19 we have

$$
\begin{aligned}
& \mathcal{H}_{-}=\bigcup_{h, N \in \mathbb{N}}\left[\bigcap_{M \geq N} B_{N, h}^{M} \cap\left(\left(C\left(\mathbb{R}^{2}\right) \times \mathbb{R}^{2}\right) \backslash A_{N, h}^{-}\right)\right], \\
& \mathcal{H}_{+}=\bigcup_{h, N \in \mathbb{N}}\left[\bigcap_{M \geq N} B_{N, h}^{M} \cap\left(\left(C\left(\mathbb{R}^{2}\right) \times \mathbb{R}^{2}\right) \backslash A_{N, h}^{+}\right)\right],
\end{aligned}
$$

and

$$
\mathcal{H}_{\infty}=\bigcap_{M \in \mathbb{N}}\left(A_{M}^{+} \cap A_{M}^{-}\right)
$$

Therefore $\mathcal{H}_{-}$and $\mathcal{H}_{+}$are $\boldsymbol{\Sigma}_{4}^{0}$, while $\mathcal{H}_{\infty}$ is $\boldsymbol{\Pi}_{3}^{0}$.

By modifying the proof of Lemma 5.7 (notice that in that proof if $\alpha \in S_{4}$ then $\left.\left(F_{\alpha},-1,0\right) \in \mathcal{H}_{+}\right)$it is not difficult to show that $\mathcal{H}_{\infty}$ is actually $\mathbf{\Pi}_{3^{-}}^{0}$ hard, and hence $\boldsymbol{\Pi}_{3}^{0}$-complete. It suffices to delete $\chi$ and the various $\chi^{n}$ 's with $n>0$ and flip the behavior of $F_{\alpha}$ inside the $T_{n}$ 's with $n$ odd so that $\lim _{+} \psi^{2 k+1}=+\infty$. This construction yields $\left(F_{\alpha},-1,0\right) \in \mathcal{H}_{\infty}$ if and only if $\forall n \forall^{\infty} m \alpha(n, m)=0$.

TheOREM 5.21. $\mathcal{G}$ is $\boldsymbol{\Sigma}_{4}^{0}$-complete.

Pr o of. The $\boldsymbol{\Sigma}_{4}^{0}$-hardness of $\mathcal{G}$ was proved in Lemma 5.7. Since the finite union of $\boldsymbol{\Sigma}_{4}^{0}$ and $\boldsymbol{\Pi}_{3}^{0}$ sets is $\boldsymbol{\Sigma}_{4}^{0}$, Theorem 5.18 and Lemma 5.20 show that $\mathcal{G}^{+}$is $\boldsymbol{\Sigma}_{4}^{0}$. But then $\mathcal{G}^{-}$is also $\boldsymbol{\Sigma}_{4}^{0}$ and $\mathcal{G}=\mathcal{G}^{+} \cap \mathcal{G}^{-}$is $\boldsymbol{\Sigma}_{4}^{0}$.

\section{References}

[1] H. Becker, Descriptive set theoretic phenomena in analysis and topology, in: Set Theory of the Continuum, H. Judah, W. Just and H. Woodin (eds.), Math. Sci. Res. Inst. Publ. 26, Springer, 1992, 1-25.

[2] G. Beer, Topologies on Closed and Closed Convex Sets, Kluwer, 1993.

[3] L. Faina, Uniqueness and continuous dependence of the solutions for functional differential equations as a generic property, Nonlinear Anal. 23 (1994) 745-754.

[4] A. Kanamori, The emergence of descriptive set theory, in: Essays on the Development of the Foundations of Mathematics, J. Hintikka (ed.), Kluwer, 1995, 241-262.

[5] A. S. Kechris, Classical Descriptive Set Theory, Springer, 1995.

[6] A. Lasota and J. A. Yorke, The generic property of existence of solutions of differential equations in Banach space, J. Differential Equations 13 (1973), 1-12. 
[7] Y. N. Moschovakis, Descriptive Set Theory, North-Holland, 1980.

[8] W. Orlicz, Zur Theorie der Differentialgleichung $y^{\prime}=f(x, y)$, Bull. Internat. Acad. Polon. Sci. Lettres Sér. A Sci. Math. 1932, 221-228.

[9] S. G. Simpson, Which set existence axioms are needed to prove the Cauchy/Peano theorem for ordinary differential equations?, J. Symbolic Logic 49 (1984), 783-802.

Dipartimento di Matematica Università di Torino

via Carlo Alberto 10

10123 Torino, Italy

E-mail: andretta@dm.unito.it marcone@dm.unito.it

Received 15 September 1996;

in revised form 15 January 1997 